\title{
On Lagrange's four squares theorem with almost prime variables
}

\author{
By Kai-Man Tsang at Hong Kong and Lilu Zhao at Hefei
}

\begin{abstract}
In 1994, Brüdern and Fouvry [1] initiated the investigation of Lagrange's four squares theorem with almost prime variables. In this paper, we prove that every sufficiently large integer, congruent to 4 modulo 24 , can be represented as a sum of four squares of integers, each of which has at most four prime factors. Instead of the four-dimensional vector sieve developed by Brüdern and Fouvry [1], we establish this result by combining the threedimensional sieve and the switching principle.
\end{abstract}

\section{Introduction}

We consider the equation of Lagrange

$$
x_{1}^{2}+x_{2}^{2}+x_{3}^{2}+x_{4}^{2}=N
$$

with multiplicative restrictions. It is expected that sufficiently large integers under certain necessary congruence condition can be written as sums of four squares of primes. This problem has not been solved so far. However Hua [10] proved that all large integers congruent to 5 modulo 24 are sums of five squares of primes by using Vinogradov's method for the ternary Goldbach problem.

Kloosterman [11] developed the circle method to study the asymptotic formula for the number of integer solutions of the following positive definite quadratic forms

$$
a_{1} x_{1}^{2}+a_{2} x_{2}^{2}+a_{3} x_{3}^{2}+a_{4} x_{4}^{2}=N .
$$

Estermann [5] investigated the indefinite quadratic forms via the circle method and the Kloosterman refinement. The classical circle method with mean value theorems (see [16] for the exposition) provides an asymptotic formula for quadratic forms with five or more variables only.

The work described in this paper was substantially supported by a grant from the Research Grants Council of the Hong Kong Special Administrative Region, China (HKU 705610P). The second author is also supported by the National Natural Science Foundation of China (Grant No. 11401154). 
Greaves [6] considered the solutions of (1.1) with two prime and two integral variables. Plaksin [12] and Shields [14] obtained an asymptotic formula for the number of solutions. Podsypanin [13] derived an asymptotic formula for the number of solutions of (1.1) in which $x_{1}, x_{2}, x_{3}, x_{4}$ are square-free.

In 1994, Brüdern and Fouvry [1] established that every sufficiently large integer, congruent to 4 modulo 24, can be written as the sum of four squares of integers, each of which has at most 34 prime factors.

In 2003, Heath-Brown and Tolev [9] managed to solve the equation

$$
p^{2}+x_{1}^{2}+x_{2}^{2}+x_{3}^{2}=N
$$

with multiplicative restrictions, where $p$ denotes a prime number. Precisely, they established the solvability of (1.2) with each of $x_{i}$ having at most 101 prime divisors. This was improved by Tolev [15] who showed that 101 can be replaced by 80, and then improved by Cai [3] who showed that 42 is acceptable. Our first result is as follows.

Theorem 1.1. Every sufficiently large integer $N$, congruent to 4 modulo 24 , can be represented in the form of

$$
N=p^{2}+x_{1}^{2}+x_{2}^{2}+x_{3}^{2},
$$

where $p$ is a prime and each of $x_{1}, x_{2}, x_{3}$ has at most five prime factors.

As in [9], the proof of the above theorem will be finished in two steps. In the first step, we combine the circle method with Kloosterman refinement and the square sieve to control the reminder term uniformly for certain level $D$. In the second step, we use the sieve method to produce the almost primes. For the uniform estimate, we shall develop the strategy of Heath-Brown and Tolev, and push their method further.

Comparing to the proof in [9], two things are different. We start from applying Cauchy's inequality for summation over $k$ only rather than summations over $k$ and $\mathbf{d}$ (see (5.2) in Section 5). This subtle difference contributes to a better result because it can be proved that the error term is dominated by diagonal contributions up to $P^{\varepsilon}$ (see Lemma 12 below). To this end, we have to introduce the condition (2.8) at the beginning, and then we remove it before we close the proof. This obstacle is one of the reasons that force Brüdern and Fouvry to develop the vector sieve (cf. the discussion in [1, Section III]). Finally instead of the vector sieve which was used by Brüdern and Fouvry, and by Heath-Brown and Tolev, we appeal to the switching principle of Chen [4] to reduce the number of prime factors for each variable.

The square sieve of Heath-Brown [8] plays an important role in the proof. However we will choose the parameter $R$ to be of type $P^{\varepsilon}$. Therefore, the square sieve is really responsible for the success of the proof, while it does not determine the quality of the level $D$.

Concerning the Lagrange equation with four almost prime variables, the value 34 due to Brüdern and Fouvry was sharpened by Heath-Brown and Tolev [9] to 25, by Tolev [15] to 20, and by Cai [3] to 13. We shall prove the following result.

Theorem 1.2. Every sufficiently large integer $N$, congruent to 4 modulo 24 , can be represented in the form of

$$
N=x_{1}^{2}+x_{2}^{2}+x_{3}^{2}+x_{4}^{2},
$$

where each of $x_{1}, x_{2}, x_{3}, x_{4}$ has at most four prime factors. 
In prior works [1,9], in order to obtain a sharp result involving four almost prime variables, people considered the equation

$$
N=d_{1}^{2} x_{1}^{2}+d_{2}^{2} x_{2}^{2}+d_{3}^{2} x_{3}^{2}+d_{4}^{2} x_{4}^{2}
$$

Our approach to Theorem 1.2 is different. We consider the equation

$$
N=q^{2}+d_{1}^{2} x_{1}^{2}+d_{2}^{2} x_{2}^{2}+d_{3}^{2} x_{3}^{2},
$$

where $q$ is an almost prime. Therefore, instead of the vector sieve developed by Brüdern and Fouvry [1], we shall combine the three-dimensional sieve and the switching principle to establish Theorem 1.2.

As usual, we write $e(z)$ for $e^{2 \pi i z}$. We use $\varepsilon$ to denote a sufficiently small positive number, and the letter $A$ denotes a sufficiently large constant. We use « to denote Vinogradov's well-know notation, while implicit constants may depend on $\varepsilon$ and $A$.

\section{A crucial proposition}

Before giving the main propositions, we introduce some notations. Let $N$ be a sufficiently large integer satisfying $N \equiv 4(\bmod 24)$. Set $P=N^{1 / 2}$. The letter $p$ is reserved for prime numbers. As usual, $\mu(n), \phi(n)$ and $\tau(n)$ denote the Möbius function, Euler's totient function, and the divisor function respectively. For the natural number $q$ and real number $\alpha$, we write $e_{q}(\alpha)=e(\alpha / q)$. We use $\sum_{x(q)}$ and $\sum_{x(q)^{*}}$ to denote sums with $x$ running over a complete system, respectively reduced system of residues modulo $q$. The Gauss sums $S(q, m, n)$ and $T(q, a)$ are defined by

$$
\begin{aligned}
S(q, m, n) & =\sum_{x(q)} e_{q}\left(m x^{2}+n x\right), \quad S(q, m)=S(q, m, 0), \\
T(q, a) & =\sum_{x(q)^{*}} e_{q}\left(a x^{2}\right),
\end{aligned}
$$

and

$$
S_{\mathbf{d}}(q, m, \mathbf{n})=\prod_{j=1}^{3} S\left(q, m d_{j}^{2}, n_{j}\right), \quad S_{\mathbf{d}}(q, m)=S_{\mathbf{d}}(q, m, \mathbf{0}),
$$

where we use the bold style letter $\mathbf{d}$ to indicate the three-dimensional vectors $\left(d_{1}, d_{2}, d_{3}\right)$. Now we define the singular series

$$
\Sigma_{0}(\mathbf{d}, N)=\sum_{q=1}^{\infty} h_{\mathbf{d}}(q)=\prod_{p>2}\left(1+h_{\mathbf{d}}(p)\right),
$$

where

$$
h_{\mathbf{d}}(q)=q^{-3} \phi(q)^{-1} \sum_{a(q)^{*}} S_{\mathbf{d}}(q, a) T(q, a) e_{q}(-a N) .
$$

Let

$$
\omega_{0}(t)= \begin{cases}\exp \left(\frac{1}{(20 t-10)^{2}-1}\right) & \text { if } \frac{9}{20}<t<\frac{11}{20} \\ 0 & \text { otherwise }\end{cases}
$$


and denote

$$
\omega(x)=\omega_{0}\left(x P^{-1}\right), \quad \omega(\mathbf{x})=\prod_{j=1}^{3} \omega\left(x_{j}\right) .
$$

We define

$$
I(\beta, u)=\int_{-\infty}^{+\infty} \omega_{0}(x) e\left(\beta x^{2}+u x\right) d x, \quad I(\beta)=I(\beta, 0),
$$

and

$$
I_{\mathbf{d}}(\beta, \mathbf{u})=\prod_{j=1}^{3} I\left(\beta, u_{j} d_{j}^{-1}\right)
$$

Let

$$
H(t)=\int_{-\infty}^{+\infty} I^{3}(\beta) e(-t \beta) d \beta .
$$

For any $j \leq 15$, let $\mathcal{A}_{j}$ denote the set consisting of integers $q \leq P$ satisfying two restrictions:

(i) the number of prime factors of $q$ counting multiplicity is exactly $j$,

(ii) all prime factors of $q$ are greater than $P^{1 / 16}$.

In order to apply the sieve method, we study

$$
\mathscr{L}_{\mathbf{d}}(N):=\mathscr{L}_{\mathbf{d}}^{j}(N)=\sum_{\substack{q^{2}+x_{1}^{2}+x_{2}^{2}+x_{3}^{2}=N \\ q \in \mathcal{A}_{j}, d_{i} \mid x_{i}(1 \leq i \leq 3)}} \omega(q) \omega(\mathbf{x}) .
$$

Here we also attach the smooth weight $\omega(q)$ for the use of switching principle. The corresponding singular integral is defined by

$$
\mathcal{N}_{0}(N):=\mathcal{N}_{0}^{j}(N)=P \int_{0}^{P} H\left(1-\frac{x^{2}}{P^{2}}\right) \frac{\omega(x) C_{j}(x) d x}{\log x},
$$

where $C_{1}(x)=1$ and for $j \geq 2$,

$$
C_{j}(x)=\sum_{P^{1 / 16<p_{1} \leq \cdots \leq p_{j-1} \leq\left(x p_{1}^{-1} \cdots p_{j-2}^{-1}\right)^{1 / 2}}} \frac{\log x}{\left(\log x-\log \left(p_{1} \cdots p_{j-1}\right)\right) p_{1} \cdots p_{j-1}} .
$$

The expected main term for $\mathscr{L}_{\mathbf{d}}(N)$ is $\mathcal{N}_{0}(N) \Sigma_{0}(\mathbf{d}, N)\left(d_{1} d_{2} d_{3}\right)^{-1}$. ( Here and after, we shall often suppress the dependence on $j$ when the meaning is clear from the context.) We plan to investigate

$$
\mathscr{H}(D)=\sum_{\begin{array}{c}
d_{1}, d_{2}, d_{3} \\
{\left[d_{1}, d_{2}, d_{3}\right] \leq D}
\end{array}} \beta(\mathbf{d})\left(\mathscr{L}_{\mathbf{d}}(N)-\frac{\mathcal{N}_{0}(N) \Sigma_{0}(\mathbf{d}, N)}{d_{1} d_{2} d_{3}}\right),
$$

where $\beta(\mathbf{d})=\beta\left(d_{1}, d_{2}, d_{3}\right)$ is a real function satisfying

$$
|\beta(\mathbf{d})| \leq \tau^{2}\left(d_{1}\right) \tau^{2}\left(d_{2}\right) \tau^{2}\left(d_{3}\right),
$$

and $\beta(\mathbf{d})=0$ unless

$$
\mu\left(2 d_{1}\right) \mu\left(2 d_{2}\right) \mu\left(2 d_{3}\right) \neq 0 .
$$


Let

$$
\mathscr{H}_{0}(D)=\sum_{d_{1}, d_{2}, d_{3} \leq D} \beta(\mathbf{d})\left(\mathscr{L}_{\mathbf{d}}(N)-\frac{\mathcal{N}_{0}(N) \Sigma_{0}(\mathbf{d}, N)}{d_{1} d_{2} d_{3}}\right) .
$$

Heath-Brown and Tolev established the following result.

Proposition 1 ([9]). If $D=P^{2 / 69-\varepsilon}$, then one has

$$
\mathcal{H}_{0}(D) \ll P^{2}(\log P)^{-A} \text {. }
$$

Define

$$
B\left(d_{1}, d_{2}, d_{3}, d_{4}, N\right)=\sum_{\substack{x_{1}^{2}+x_{2}^{2}+x_{3}^{2}+x_{4}^{2}=N \\ x_{i} \equiv 0\left(\bmod d_{i}\right)}} \prod_{i=1}^{4} \omega\left(x_{i}\right)
$$

and

$$
\Sigma_{1}\left(d_{1}, d_{2}, d_{3}, d_{4}, N\right)=\sum_{q=1}^{\infty} q^{-4} \sum_{a(q)^{*}} e_{q}(-a N) \prod_{i=1}^{4} S\left(q, a d_{i}^{2}\right)
$$

Let

$$
\mathscr{H}_{0}^{*}(D)=\sum_{d_{1}, d_{2}, d_{3}, d_{4} \leq D}\left|B\left(d_{1}, d_{2}, d_{3}, d_{4}, N\right)-\kappa_{1} N \frac{\Sigma_{1}\left(d_{1}, d_{2}, d_{3}, d_{4}, N\right)}{d_{1} d_{2} d_{3} d_{4}}\right|,
$$

where the constant $\kappa_{1}$ is given by

$$
\kappa_{1}=\int_{-\infty}^{\infty} I^{4}(\beta) e(-\beta) d \beta
$$

It was proved by Heath-Brown and Tolev that:

Proposition 2 ([9]). If $D \leq P^{1 / 8-\varepsilon}$, then one has

$$
\mathscr{H}_{0}^{*}(D) \ll P^{2-\varepsilon} \text {. }
$$

Proposition 2 improves upon the result of Brüdern and Fouvry [1] who essentially showed $\mathscr{H}_{0}^{*}(D) \ll P^{2-\varepsilon}$ provided that $D \leq P^{1 / 11-\varepsilon}$.

Concerning $\mathscr{H}(D)$, we prove the following result.

Proposition 3. Suppose that $D<P^{1 / 2-\varepsilon}$. Then we have

$$
\mathscr{H}(D) \ll P^{2}(\log P)^{-A} .
$$

Remark. (i) The function $\beta$ (d) may depend on $N$, but in view of (2.6), the absolute value of $\beta(\mathbf{d})$ is bounded from above by $\prod_{j=1}^{3} \tau^{2}\left(d_{j}\right)$ which is independent of $N$.

(ii) With the application to Lagrange's four squares theorem with almost prime variables in mind, Proposition 3 improves upon both Proposition 1 and Proposition 2.

In order to prove Proposition 1, Heath-Brown and Tolev investigated the following object:

$$
\Omega_{\mathbf{d}}(n)=\sum_{\substack{x_{1}^{2}+x_{2}^{2}+x_{3}^{2}=n \\ d_{j} \mid x_{j}(1 \leq j \leq 3)}} \omega(\mathbf{x})
$$


The application of circle method suggests that the sum $\Omega_{\mathbf{d}}(n)$ may be approximated by

$$
\mathcal{M}_{\mathbf{d}, Q}(n)=\frac{P H\left(n N^{-1}\right)}{d_{1} d_{2} d_{3}} \sum_{q \leq Q} q^{-3} \sum_{a(q)^{*}} e_{q}(-a n) S_{\mathbf{d}}(q, a) .
$$

Consider

$$
\widetilde{\mathcal{E}}(D, Q)=\sum_{d_{1}, d_{2}, d_{3} \leq D} \tau\left(d_{1}\right) \tau\left(d_{2}\right) \tau\left(d_{3}\right) \sum_{k \leq P}\left|\Omega_{\mathbf{d}}\left(N-k^{2}\right)-\mathcal{M}_{\mathbf{d}, Q}\left(N-k^{2}\right)\right| .
$$

Heath-Brown and Tolev established

$$
\widetilde{\mathcal{E}}(D, Q) \ll P^{2-\varepsilon}
$$

provided that $Q=P^{20 / 23}$ and $D=P^{2 / 69-10 \varepsilon}$. Our purpose is to establish the following result.

Proposition 4. Let

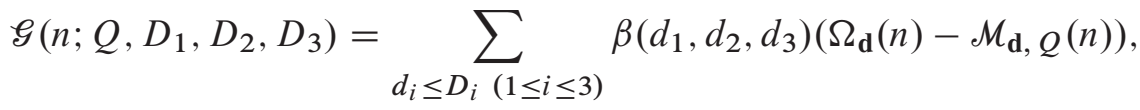

where $\beta\left(d_{1}, d_{2}, d_{3}\right)$ satisfies (2.6), (2.7) and

$$
\left(d_{i}, d_{j}\right) \leq P^{\varepsilon} \quad \text { for } 1 \leq i<j \leq 3 .
$$

Suppose that $D_{1} D_{2} D_{3}<P^{1 / 2-6 \varepsilon}$ and $P^{1-4 \varepsilon}<Q<P^{1-2 \varepsilon}$. Then we have

$$
\sum_{k \leq P}\left|\mathcal{E}\left(N-k^{2} ; Q, D_{1}, D_{2}, D_{3}\right)\right| \ll P^{2-\varepsilon} .
$$

Proposition 4 will be proved in Sections 3-6. As an application of Proposition 4, we shall prove Proposition 3 in Section 7.

\section{Basic assumptions}

We first quote some lemmas which are well known.

Lemma 1. The Gauss sum defined by (2.1) satisfies:

(i) If $\left(q_{1}, q_{2}\right)=1$, then

$$
S\left(q_{1} q_{2}, a_{1} q_{2}+a_{2} q_{1}, n\right)=S\left(q_{1}, a_{1} q_{2}^{2}, n\right) S\left(q_{2}, a_{2} q_{1}^{2}, n\right) .
$$

(ii) Suppose that $(q, m)=k$. Then we have

$$
S(q, m, n)= \begin{cases}k S(q / k, m / k, n / k) & \text { if } k \mid n, \\ 0 & \text { otherwise. }\end{cases}
$$


(iii) If $(q, 2 m)=1$, then

$$
S(q, m, n)=e_{q}\left(-\overline{4 m} n^{2}\right)\left(\frac{m}{q}\right) S(q, 1),
$$

where $\bar{x}$ denotes the inverse of $x$ modulo $q$.

(iv) If $q$ is odd, then

$$
S(q, 1)= \begin{cases}q^{1 / 2} & \text { if } q \equiv 1(\bmod 4) \\ i q^{1 / 2} & \text { if } q \equiv 3(\bmod 4)\end{cases}
$$

(v) If $(2, a)=1$, then

$$
\left|S\left(2^{l}, a, n\right)\right| \leq 2^{1+l / 2} .
$$

(vi) For any odd integer $q$, we have $|\gamma(q)| \leq q^{1 / 2}$, where

$$
\gamma(q)=\sum_{x(q)^{*}}\left(\frac{x}{q}\right) e_{q}(x) .
$$

Throughout, by $\left(\frac{l}{q}\right)$ we denote the Jacobi symbol.

Lemma 2. Denote the Kloosterman sum by

$$
K(p, m, n)=\sum_{x=1}^{p-1} e_{p}(m x+n \bar{x})
$$

If $p \nmid(m, n)$, then

$$
|K(p, m, n)| \leq 2 p^{1 / 2} .
$$

Lemma 3. Let $c_{q}(n)$ be the Ramanujan sum given by

$$
c_{q}(n)=\sum_{x(q)^{*}} e_{q}(x n) .
$$

Then we have

$$
\left|c_{q}(n)\right| \leq(q, n)
$$

Lemma 4. The following statements hold.

(i) Suppose that $u=\max \left\{\left|u_{1}\right|, \ldots,\left|u_{6}\right|\right\}>0$. Then we have

$$
\int_{-\infty}^{+\infty}\left|I\left(\beta, u_{1}\right) \cdots I\left(\beta, u_{6}\right)\right| d \beta \ll \min \left\{1, u^{-2+\varepsilon}\right\} .
$$

(ii) Suppose that $\beta_{0}>0$. Then we have

$$
\int_{|\beta| \geq \beta_{0}}|I(\beta)|^{6} d \beta \ll \beta_{0}^{-2} .
$$

Lemma 4 is a consequence of [9, Lemma 9 and Lemma 10]. Let

$$
f_{d}(\alpha)=\sum_{\substack{x \in \mathbb{Z} \\ x \equiv 0(\bmod d)}} \omega(x) e\left(\alpha x^{2}\right), \quad f_{\mathbf{d}}(\alpha)=\prod_{i=1}^{3} f_{d_{i}}(\alpha),
$$


and let

$$
\mathcal{W}_{\mathbf{d}, Q}(\alpha)=\sum_{n \in \mathbb{Z}} \mathcal{M}_{\mathbf{d}, Q}(n) e(\alpha n)
$$

The following two lemmas provide useful approximations to $f_{d}(\alpha)$ and $W_{\mathbf{d}, Q}(\alpha)$ respectively.

Lemma 5 ([9, Lemma 12]). Let $q, d, b, h \in \mathbb{N}$, let $\beta \in \mathbb{R}, q \leq P$ with $|\beta| \leq(q P)^{-1}$ and $d, b \leq P^{2}$. Then we have

$$
f_{d}\left(\frac{h}{b}+\beta\right)=\frac{P}{b d} \sum_{|n| \leq b d q^{-1} P^{\varepsilon}} S\left(b, h d^{2}, n\right) I\left(\beta N,-\frac{P n}{b d}\right)+O\left(P^{-A}\right),
$$

where the implicit constant depends on $A$ and $\varepsilon$.

Lemma 6 ([9, Lemma 16]). Suppose that $Q \leq P^{1-\varepsilon},|\beta| \leq(q P)^{-1}$ and $(a, q)=1$. Then we have

$$
W_{\mathbf{d}, Q}(\alpha)= \begin{cases}\frac{P^{3}}{q^{3} d_{1} d_{2} d_{3}} S_{\mathbf{d}}(q, a) I^{3}(\beta N)+O\left(P^{-A}\right) & \text { if } 1 \leq q \leq Q, \\ O\left(P^{-A}\right) & \text { if } Q<q \leq P,\end{cases}
$$

where the implicit constants depend on $A$ and $\varepsilon$.

Set

$$
\sigma_{0}=\int_{-\infty}^{+\infty}\left|I^{6}(\beta)\right| d \beta
$$

Lemma 7. Let

$$
B(\alpha)=\sum_{n \in \mathbb{Z}} H^{2}\left(\frac{n}{N}\right) e(n \alpha)
$$

where $H(t)$ is given in (2.3). We have

$$
B(\alpha)= \begin{cases}\sigma_{0} P^{2}+O\left(P^{-A}\right) & \text { if } \alpha \in \mathbb{Z}, \\ O\left(P^{-A}\right) & \text { if }\|\alpha\| \geq P^{\varepsilon-2},\end{cases}
$$

where the implicit constants may depend on $A$ and $\varepsilon$.

Lemma 7 is [9, formula (115)].

\section{Some preparations}

Let

$$
\theta\left(q ; \mathbf{d}, \mathbf{t}, \mathbf{n}, \mathbf{l}, v, b_{1}, b_{2}\right)=\sum_{a(q)^{*}} e_{q}\left(\overline{a b_{1}} v\right) S_{\mathbf{d}}\left(q, a b_{2}^{2}, \mathbf{n}\right) S_{\mathbf{t}}\left(q,-a b_{1}^{2},-\mathbf{l}\right)
$$

and let

$$
\eta=\eta\left(\mathbf{d}, \mathbf{t}, \mathbf{n}, \mathbf{l}, v, b_{1}, b_{2}\right)=b_{1}^{2} b_{2}^{2}\left(-4 \frac{v}{b_{1}}+\sum_{\substack{j=1 \\ n_{j} \neq 0}}^{3} \frac{n_{j}^{2}}{d_{j}^{2} b_{2}^{2}}-\sum_{\substack{i=1 \\ l_{i} \neq 0}}^{3} \frac{l_{i}^{2}}{t_{i}^{2} b_{1}^{2}}\right) \times 1 \mathrm{~cm},
$$


where we use lcm to denote the least common multiple of $d_{j}(j \in J)$ and $t_{i}(i \in I)$ with the index sets $J=\left\{1 \leq j \leq 3: n_{j} \neq 0\right\}$ and $I=\left\{1 \leq i \leq 3: l_{i} \neq 0\right\}$. For $\mathbf{n}=\mathbf{l}=\mathbf{0} \in \mathbb{Z}^{3}$, we assume $1 \mathrm{~cm}=1$. For simplicity, in this section we always assume that $\mathbf{d}$ satisfies (2.7), (2.8) and $d_{i} \leq D_{i}$ for $1 \leq i \leq 3$, and we also have analogous assumptions with $\mathbf{t}$ in place of $\mathbf{d}$. We use the notations $(q, \mathbf{k})=\left(q, k_{1}\right)\left(q, k_{2}\right)\left(q, k_{3}\right)$ and $\left(q, \mathbf{k}^{2}\right)=\left(q, k_{1}^{2}\right)\left(q, k_{2}^{2}\right)\left(q, k_{3}^{2}\right)$ for $\mathbf{k}=\mathbf{d}$ and $\mathbf{t}$.

Lemma 8. Let $\theta(q):=\theta\left(q ; \mathbf{d}, \mathbf{t}, \mathbf{n}, \mathbf{l}, v, b_{1}, b_{2}\right)$ be defined in formula (4.1). Suppose that $(2, r)=1$ and $\left(b_{1} b_{2}, 2 r\right)=1$. Then we have

$$
\left|\theta\left(2^{u} r\right)\right| \leq \begin{cases}2^{4 u+6} r^{3}(r, \eta)(r, \mathbf{d})(r, \mathbf{t}) & \text { if } \eta \neq 0 \text { and }(\mathbf{n}, \mathbf{l}) \neq \mathbf{0} \in \mathbb{Z}^{6}, \\ 2^{4 u+6} r^{4}\left(r, \mathbf{d}^{2}\right)^{1 / 2}\left(r, \mathbf{t}^{2}\right)^{1 / 2} & \text { otherwise, }\end{cases}
$$

where $\eta=\eta\left(\mathbf{d}, \mathbf{t}, \mathbf{n}, \mathbf{l}, v, b_{1}, b_{2}\right)$ is given by (4.2).

Proof. As a function of $q, \theta(q)=\theta\left(q ; \mathbf{d}, \mathbf{t}, \mathbf{n}, \mathbf{l}, v, b_{1}, b_{2}\right)$ is multiplicative. So it suffices to prove

$$
\left|\theta\left(2^{u}\right)\right| \leq 2^{4 u+6}
$$

and

$$
\left|\theta\left(p^{\alpha}\right)\right| \leq \begin{cases}p^{3 \alpha}\left(p^{\alpha}, \eta\right)\left(p^{\alpha}, \mathbf{d}\right)\left(p^{\alpha}, \mathbf{t}\right) & \text { if } \eta \neq 0 \text { and }(\mathbf{n}, \mathbf{l}) \neq \mathbf{0}, \\ p^{4 \alpha}\left(p^{\alpha}, \mathbf{d}^{2}\right)^{1 / 2}\left(p^{\alpha}, \mathbf{t}^{2}\right)^{1 / 2} & \text { otherwise, }\end{cases}
$$

for odd prime $p$. Plainly, (4.3) follows from Lemma 1 (v). By Lemma 1 (ii) and (iii),

$$
S\left(p^{\alpha}, a m^{2}, n\right)= \begin{cases}e\left(\frac{-\overline{4 m^{2} a} n^{2}}{p^{\alpha}}\right) S\left(p^{\alpha}, a\right) & \text { if } p \nmid m, \\ e\left(\frac{-4 s^{2} a t^{2}}{p^{\alpha-2}}\right) p^{2} S\left(p^{\alpha-2}, a\right) & \text { if } p \| m=p s, p^{2} \mid n=p^{2} t \text { and } \alpha \geq 2, \\ 0 & \text { if } p \| m=p s, p^{2} \nmid n \text { and } \alpha \geq 2, \\ p & \text { if } p|m, p| n \text { and } \alpha=1, \\ 0 & \text { if } p \mid m, p \nmid n \text { and } \alpha=1,\end{cases}
$$

where $p \| m$ means $p \mid m$ but $p^{2} \nmid m$. Then for $\alpha \geq 2$, we have

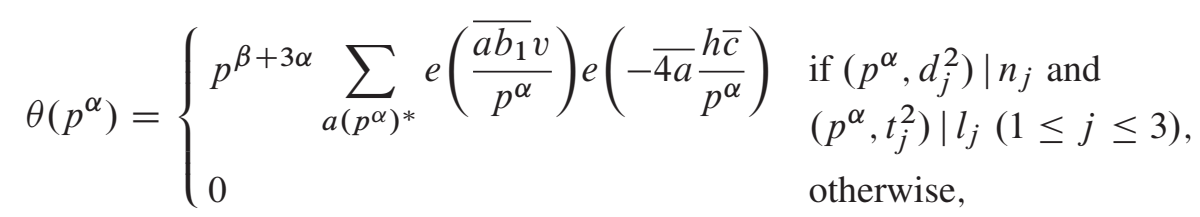

where $p^{\beta} \| d_{1} d_{2} d_{3} t_{1} t_{2} t_{3}$ and

$$
\frac{h}{c}=\sum_{j=1}^{3}\left(\frac{n_{j}^{2}}{d_{j}^{2} b_{2}^{2}}-\frac{l_{j}^{2}}{t_{j}^{2} b_{1}^{2}}\right)
$$

with $(h, c)=1$. This proves (4.4) in the case $\alpha \geq 2$ by using Lemma 3. Hence we turn to the case $\alpha=1$. 
If $p \nmid d_{1} d_{2} d_{3} t_{1} t_{2} t_{3}$, then formula (4.5) still holds for $\alpha=1$. We therefore confine to the case $p \mid d_{1} d_{2} d_{3} t_{1} t_{2} t_{3}$. Note that

$$
\left|S\left(p, a m^{2}, n\right)\right| \leq p^{1 / 2}(p, m)^{1 / 2}
$$

by Lemma 1 (ii), (iii) and (iv). The second estimate of (4.4) follows easily. Now we assume that $\eta \neq 0$ and $(\mathbf{n}, \mathbf{l}) \neq \mathbf{0}$. If $p \| d_{1} d_{2} d_{3} t_{1} t_{2} t_{3}$, then $\theta(p)$ is in the form

$$
\theta(p)=p S(p, 1)^{5} \sum_{a(p)^{*}}\left(\frac{a}{p}\right) e\left(\frac{\overline{a b} c}{q}\right),
$$

where $(b, p)=1$. The summation over $a$ is either 0 or a Gauss sum. So, by Lemma 1 (vi), we have

$$
|\theta(p)| \leq p^{3+1 / 2}(p, \mathbf{d})^{1 / 2}(p, \mathbf{t})^{1 / 2}=p^{3}(p, \mathbf{d})(p, \mathbf{t}) .
$$

If $p^{2} \mid d_{1} d_{2} d_{3} t_{1} t_{2} t_{3}$, then we apply the trivial bound for summation over $a$ to get

$$
|\theta(p)| \leq p^{4}(p, \mathbf{d})^{1 / 2}(p, \mathbf{t})^{1 / 2} \leq p^{3}(p, \mathbf{d})(p, \mathbf{t}) .
$$

The proof is completed.

From now on, we assume that $p, p^{\prime}$ are two different primes satisfying $R \leq p, p^{\prime}<2 R$ and $\left(p p^{\prime}, d_{1} d_{2} d_{3} t_{1} t_{2} t_{3} N\right)=1$. For $\Delta \mid\left(p p^{\prime}\right)^{2}$, we have the unique decomposition $\Delta=\delta \delta^{\prime}$, where $\left(p, \delta^{\prime}\right)=\left(p^{\prime}, \delta\right)=1$. We use $\pi$ and $\pi^{\prime}$ to denote a power of $p$, and respectively a power of $p^{\prime}$ (note that $\pi$ and $\pi^{\prime}$ may be equal to 1 ).

Let us define

$$
\begin{aligned}
W\left(\Delta, q ; \mathbf{d}, \mathbf{t}, \mathbf{n}, \mathbf{l}, p, p^{\prime}, v\right)=\sum_{s\left(p p^{\prime}\right)^{*}} & \left(\frac{s}{p p^{\prime}}\right) e_{p p^{\prime}}(-s N) \sum_{\substack{a(q)^{*} \\
\left(a p p^{\prime}+s q, q p p^{\prime}\right)=\Delta}} e_{q}(\bar{a} v) \\
& \times S_{\mathbf{d}}\left(q p p^{\prime} \Delta^{-1},\left(a p p^{\prime}+s q\right) \Delta^{-1}, \mathbf{n}\right) S_{\mathbf{t}}(q,-a,-\mathbf{l})
\end{aligned}
$$

and

$$
\begin{aligned}
\mathcal{R}\left(\delta, p, \pi ; \mathbf{d}, \mathbf{t}, \mathbf{n}, \mathbf{l}, m_{1}, m_{2}, n\right)=\sum_{c(p)^{*}}\left(\frac{c}{p}\right) e_{p}(-c n) \sum_{\begin{array}{c}
b(\pi)^{*} \\
(b p+c \pi, \pi p)=\delta
\end{array}} e_{\pi}\left(\overline{b m_{1}} v\right) \\
\times S_{\mathbf{d}}\left(\frac{\pi p}{\delta}, \frac{b p+c \pi}{\delta} m_{2}^{2}, \mathbf{n}\right) S_{\mathbf{t}}\left(\pi,-b m_{1}^{2},-\mathbf{l}\right) .
\end{aligned}
$$

Lemma 9. Suppose that $q=\pi \pi^{\prime} r$ with $\left(p p^{\prime}, r\right)=1$. Then one has

$$
\begin{aligned}
W\left(\Delta, q ; \mathbf{d}, \mathbf{t}, \mathbf{n}, \mathbf{l}, p, p^{\prime}, v\right)=\left(\frac{p}{p^{\prime}}\right) & \left(\frac{p^{\prime}}{p}\right) \theta\left(r ; \mathbf{d}, \mathbf{t}, \mathbf{n}, \mathbf{l}, v, \pi \pi^{\prime}, \pi \pi^{\prime} p p^{\prime} \Delta^{-1}\right) \\
& \times \mathcal{R}\left(\delta, p, \pi ; \mathbf{d}, \mathbf{t}, \mathbf{n}, \mathbf{l}, r \pi^{\prime}, \frac{r p^{\prime} \pi^{\prime}}{\delta^{\prime}}, p^{\prime} N\right) \\
& \times \mathcal{R}\left(\delta^{\prime}, p^{\prime}, \pi^{\prime} ; \mathbf{d}, \mathbf{t}, \mathbf{n}, \mathbf{l}, r \pi, \frac{r p \pi}{\delta}, p N\right) .
\end{aligned}
$$


Proof. Let

$$
a=\alpha \pi \pi^{\prime}+b \pi^{\prime} r+b^{\prime} \pi r
$$

with $(\alpha, r)=(b, p)=\left(b^{\prime}, p^{\prime}\right)=1$ and

$$
s=c p^{\prime}+c^{\prime} p
$$

with $(c, p)=\left(c^{\prime}, p^{\prime}\right)=1$. Note that

$$
\left(a p p^{\prime}+s q, q p p^{\prime}\right)=\Delta \Longleftrightarrow(b p+c \pi, p \pi)=\delta \text { and }\left(b^{\prime} p^{\prime}+c^{\prime} \pi^{\prime}, p^{\prime} \pi^{\prime}\right)=\delta^{\prime} .
$$

Obviously

$$
\frac{a p p^{\prime}+s q}{\Delta}=\alpha \frac{p \pi}{\delta} \frac{p^{\prime} \pi^{\prime}}{\delta^{\prime}}+\frac{b p+c \pi}{\delta} \frac{p^{\prime} \pi^{\prime}}{\delta^{\prime}} r+\frac{b^{\prime} p^{\prime}+c^{\prime} \pi^{\prime}}{\delta^{\prime}} \frac{p \pi}{\delta} r .
$$

With the help of Lemma 1 (i), the desired result can be obtained by changing variables.

Lemma 10. We have

$$
\begin{aligned}
& \left|\mathcal{R}\left(\delta, p, \pi ; \mathbf{d}, \mathbf{t}, \mathbf{n}, \mathbf{l}, r \pi^{\prime}, \frac{r p^{\prime} \pi^{\prime}}{\delta^{\prime}}, p^{\prime} N\right) \mathcal{R}\left(\delta^{\prime}, p^{\prime}, \pi^{\prime} ; \mathbf{d}, \mathbf{t}, \mathbf{n}, \mathbf{l}, r \pi, \frac{r p \pi}{\delta}, p N\right)\right| \\
& \leq \min \left\{\Delta^{-2} R^{4}\left(4 \pi \pi^{\prime}\right)^{5}\left(1+\Delta R^{-2}\right)^{-1}, 2^{6} R^{4}\left(\pi \pi^{\prime}\right)^{4}\right\} .
\end{aligned}
$$

Proof. We can assume that $\delta \mid p \pi$ and $\delta \mid p^{\prime} \pi^{\prime}$. Otherwise, the desired estimate holds trivially. We write

$$
\mathcal{R}(\delta, p, \pi):=\mathcal{R}\left(\delta, p, \pi ; \mathbf{d}, \mathbf{t}, \mathbf{n}, \mathbf{l}, r \pi^{\prime}, \frac{r p^{\prime} \pi^{\prime}}{\delta^{\prime}}, p^{\prime} N\right) .
$$

Obviously $\left(1+\delta R^{-1}\right)^{-1}\left(1+\delta^{\prime} R^{-1}\right)^{-1} \leq\left(1+\Delta R^{-2}\right)^{-1}$. Thus it is enough to prove

$$
|\mathcal{R}(\delta, p, \pi)| \leq \min \left\{\delta^{-2} R^{2}(2 \pi)^{5}\left(1+\delta R^{-1}\right)^{-1}, 8 R^{2} \pi^{4}\right\} .
$$

Set $m_{1}=r \pi^{\prime}$ and $m_{2}=r p^{\prime} \pi^{\prime} / \delta^{\prime}$. It is clear that $p \nmid m_{1} m_{2}$.

When $\delta=1,(b p+c \pi, \pi p)=\delta$ is equivalent to $\pi=1$. So one has

$$
\begin{aligned}
\mathcal{R}(1, p, \pi) & =\sum_{c(p)^{*}}\left(\frac{c}{p}\right) e_{p}\left(-c p^{\prime} N\right) S_{\mathbf{d}}\left(p, c m_{2}^{2}, \mathbf{n}\right) \\
& =S(p, 1)^{3} \sum_{c(p)^{*}} e_{p}\left(-c p^{\prime} N\right) e_{p}\left(-\overline{4 c m_{2}^{2}} \sum_{j=1}^{3} n_{j}^{2} \overline{d_{j}^{2}}\right) .
\end{aligned}
$$

Then by Lemma 1 (iv) and Lemma 2, we get

$$
|\mathcal{R}(1, p, \pi)| \leq 2 p^{2} .
$$

When $\delta=p$, we have $(b p+c \pi, \pi p)=\delta$ if and only if $p \mid \pi$ and $\left(b+c \frac{\pi}{p}, p\right)=1$. Hence

$$
\begin{aligned}
\mathcal{R}(\delta, p, \pi)=\sum_{c(p)^{*}}\left(\frac{c}{p}\right) e_{p}\left(-c p^{\prime} N\right) \sum_{\substack{b(\pi)^{*} \\
\left(b+c \pi p^{-1}, \pi\right)=1}} e_{\pi}\left(\overline{b m_{1}} v\right) \\
\times S_{\mathbf{d}}\left(\pi,\left(b+c \frac{\pi}{p}\right) m_{2}^{2}, \mathbf{n}\right) S_{\mathbf{t}}\left(\pi,-b m_{1}^{2},-\mathbf{l}\right) .
\end{aligned}
$$

Using the trivial bound for summations over $c$ and $b$, we get

$$
|\mathcal{R}(\delta, p, \pi)| \leq p \pi^{4} .
$$


When $\delta=p^{2}$, we have $(b p+c \pi, \pi p)=\delta$ if and only if $\pi=p$ and $b+c \equiv 0(\bmod p)$. Therefore, one has

$$
\begin{aligned}
\mathcal{R}(\delta, p, \pi) & =\sum_{c(p)^{*}}\left(\frac{c}{p}\right) e_{p}\left(-c p^{\prime} N\right) \sum_{\substack{b(p)^{*} \\
b+c \equiv 0(\bmod p)}} e_{p}\left(\overline{b m_{1}} v\right) S_{\mathbf{t}}\left(p,-b m_{1}^{2},-\mathbf{l}\right) \\
& =\sum_{c(p)^{*}}\left(\frac{c}{p}\right) e_{p}\left(-c p^{\prime} N\right) e_{p}\left(\overline{-c m_{1}} v\right) S_{\mathbf{t}}\left(p, c m_{1}^{2},-\mathbf{l}\right) \\
& =S^{3}(p, 1) \sum_{c(p)^{*}} e_{p}\left(-c p^{\prime} N\right) e_{p}\left(\overline{-c m_{1}} v\right) e_{p}\left(-\overline{4 c m_{1}^{2}} \sum_{j=1}^{3} l_{j}^{2} \overline{t_{j}^{2}}\right) .
\end{aligned}
$$

Then we get

$$
|\mathcal{R}(\delta, p, \pi)| \leq 2 p^{2} .
$$

This completes the proof.

In view of Lemma 8, we define

$$
\xi(r, \eta, \mathbf{d}, \mathbf{t})= \begin{cases}(r, \eta)(r, \mathbf{d})(r, \mathbf{t}) & \text { if } \eta \neq 0 \text { and }(\mathbf{n}, \mathbf{l}) \neq \mathbf{0}, \\ r\left(r, \mathbf{d}^{2}\right)^{1 / 2}\left(r, \mathbf{t}^{2}\right)^{1 / 2} & \text { otherwise. }\end{cases}
$$

We summarize from Lemmas 8-10 that

Lemma 11. Suppose that $q=2^{u} \pi \pi^{\prime} r$ with $\left(2 p p^{\prime}, r\right)=1$. Then we have

$$
\begin{aligned}
& \left|W\left(\Delta, q ; \mathbf{d}, \mathbf{t}, \mathbf{n}, \mathbf{l}, p, p^{\prime}, v\right)\right| \\
& \quad \leq 2^{4 u+6} r^{3} \xi(r, \eta, \mathbf{d}, \mathbf{t}) \min \left\{\Delta^{-2} R^{4}\left(4 \pi \pi^{\prime}\right)^{5}\left(1+\Delta R^{-2}\right)^{-1}, 2^{6} R^{4}\left(\pi \pi^{\prime}\right)^{4}\right\} .
\end{aligned}
$$

The following estimate is a key ingredient in our proof.

Lemma 12. Let $H>0$. Define

$$
U=\sum_{|v| \leq P} \frac{1}{1+|v|} \sum_{\mathbf{d}} \sum_{\mathbf{t}} \sum_{\substack{(\mathbf{n}, \mathbf{l}) \neq \mathbf{0} \in \mathbb{Z}^{6} \\\left|n_{j}\right| \leq d_{j} H P^{\varepsilon}, l_{i} \mid \leq t_{i} P^{\varepsilon} \\ \eta\left(\mathbf{d}, \mathbf{t}, \mathbf{n}, \mathbf{l}, v, b_{1}, b_{2}\right)=0}} \max \left\{\frac{\left|n_{j}\right|}{d_{j} H}, \frac{\left|l_{i}\right|}{t_{i}}\right\}^{-1} .
$$

Suppose that $\left(b_{1} b_{2}, 2 d_{1} d_{2} d_{3} t_{1} t_{2} t_{3}\right)=1$. Then we have

$$
u \ll P^{\varepsilon}\left(D_{1} D_{2} D_{3}\right)^{2}(1+H) .
$$

Remark. Recalling (4.2), when $b_{1}=b_{2}=1$ and $v=0$, one has the diagonal contributions from $\mathbf{d}=\mathbf{t}$ and $\mathbf{n}=\mathbf{l}$. Therefore in the case $b_{1}=b_{2}=1$ and $H=1$, we have the lower bound $\mathcal{l} \gg\left(D_{1} D_{2} D_{3}\right)^{2}$ which coincides with the upper bound (up to $P^{\varepsilon}$ ).

Proof. We decompose $\mathcal{U}$ to get

$$
U \ll P^{\varepsilon} \sup _{\substack{N_{j} \leq D_{j} H P^{\varepsilon} \\ L_{i} \leq D_{i} P^{\varepsilon} \\|v| \leq P}} U\left(N_{1}, N_{2}, N_{3}, L_{1}, L_{2}, L_{3}\right),
$$


where

$$
\mathcal{U}\left(N_{1}, N_{2}, N_{3}, L_{1}, L_{2}, L_{3}\right)=\sum_{\mathbf{d}} \sum_{\mathbf{t}} \sum_{\substack{\mathbf{n}, \mathbf{l} \\ N_{j} \leq\left|n_{j}\right| \leq 2 N_{j}, L_{i} \leq\left|l_{j}\right| \leq 2 L_{i} \\(\mathbf{n}, \mathbf{l}) \neq \mathbf{0} \in \mathbb{Z}^{6} \\ \eta:=\eta\left(\mathbf{d}, \mathbf{t}, \mathbf{n}, \mathbf{l}, v, b_{1}, b_{2}\right)=0}} \max \left\{\frac{\left|n_{j}\right|}{d_{j} H}, \frac{\left|l_{i}\right|}{t_{i}}\right\}^{-1} .
$$

Without loss of generality, we assume either $L_{1} \neq 0$ or $N_{1} \neq 0$.

We first consider the case $L_{1} \neq 0$. Let

$$
e_{i}=\prod_{\substack{p \mid t_{i} \\ p \nmid d_{1} d_{2} d_{3} t_{1} t_{2} t_{3} t_{i}^{-1}}} p
$$

Since $e_{i}^{2}$ is a divisor of $\eta-b_{1}^{2} b_{2}^{2} l_{i}^{2} /\left(t_{i}^{2} b_{1}^{2}\right) \mathrm{lcm}$ and $\left(b_{1}^{2} b_{2}^{2} t_{i}^{-2} \mathrm{lcm}, e_{i}^{2}\right)=1$, we have $e_{i}^{2} \mid l_{i}^{2}$ and then $e_{i} \mid l_{i}$. Hence for fixed $\mathbf{d}$ and $\mathbf{t}$, there are at most $2 L_{1} e_{1}^{-1}\left(1+2 L_{2} e_{2}^{-1}\right)\left(1+2 L_{3} e_{3}^{-1}\right)$ possible choices for $\mathbf{l}$. Clearly

$$
\max \left\{\frac{\left|n_{j}\right|}{d_{j} H}, \frac{\left|l_{i}\right|}{t_{i}}\right\}^{-1} \leq t_{1} L_{1}^{-1} .
$$

Now fix $\mathbf{d}, \mathbf{t}$ and $\mathbf{l}$, we claim there are at most $O\left(P^{\varepsilon}+H P^{\varepsilon}\right)$ possible choices for $n_{1}$. Suppose that $\left(n_{1}, n_{2}, n_{3}\right)$ and $\left(n_{1}^{\prime}, n_{2}^{\prime}, n_{3}^{\prime}\right)$ are two solutions for $\eta\left(\mathbf{d}, \mathbf{t}, \mathbf{n}, \mathbf{l}, v, b_{1}, b_{2}\right)=0$. Then

$$
\sum_{j=1}^{3} \frac{n_{j}^{2}-n_{j}^{\prime 2}}{d_{j}^{2}}=0
$$

We write it in the form

$$
\frac{\left[d_{1}, d_{2}, d_{3}\right]^{2}}{d_{1}^{2}}\left(n_{1}^{2}-n_{1}^{\prime 2}\right)=-\frac{\left[d_{1}, d_{2}, d_{3}\right]^{2}}{d_{2}^{2}}\left(n_{2}^{2}-n_{2}^{\prime 2}\right)-\frac{\left[d_{1}, d_{2}, d_{3}\right]^{2}}{d_{3}^{2}}\left(n_{3}^{2}-n_{3}^{\prime 2}\right) .
$$

Let

$$
k=\prod_{\substack{p \mid d_{1} \\ p \nmid d_{2} d_{3}}} p
$$

We see that $n_{1}^{2} \equiv n_{1}^{\prime 2}\left(\bmod k^{2}\right)$. So we can find $K \mid k^{2}$ with $K \geq k$ such that either $K \mid n_{1}+n_{1}^{\prime}$ or $K \mid n_{1}-n_{1}^{\prime}$. We deal with $K \mid n_{1}-n_{1}^{\prime}$, and the other case can be handled similarly. Suppose that $n_{1}-n_{1}^{\prime}=K m$ for some $m$ with $0 \leq|m| \leq d_{1} H P^{\varepsilon} K^{-1}$. Note that $K \geq d_{1} P^{-\varepsilon}$ due to $K \geq k$ and the condition (2.8). Then the number of possible choices for $m$ is $O\left((1+H) P^{\varepsilon}\right)$. The number of $K$ satisfying $K \mid k^{2}$ is at most $O\left(P^{\varepsilon}\right)$. Thus there are at most $O\left((1+H) P^{\varepsilon}\right)$ choices for $n_{1}$. Then $n_{2}, n_{3}$ can be determined (up to at most $P^{\varepsilon}$ choices) by $\mathbf{d}, \mathbf{t}, \mathbf{l}, n_{1}$ due to the equation $\eta=0$. Hence

$$
\begin{aligned}
U\left(N_{1}, N_{2}, N_{3}, L_{1}, L_{2}, L_{3}\right) & \ll \sum_{\mathbf{d}} \sum_{\mathbf{t}} \frac{L_{1}}{e_{1}}\left(1+\frac{L_{2}}{e_{2}}\right)\left(1+\frac{L_{3}}{e_{3}}\right) \frac{t_{1}}{L_{1}}(1+H) P^{\varepsilon} \\
& \ll \sum_{\mathbf{d}} \sum_{\mathbf{t}} \prod_{i=1}^{3} \frac{t_{i}}{e_{i}}(1+H) P^{\varepsilon} .
\end{aligned}
$$


Note that

$$
t_{i} \leq e_{i} \prod_{j=1}^{3}\left(d_{j}, t_{i}\right)\left(t_{1}, t_{2}\right)\left(t_{1}, t_{3}\right)\left(t_{2}, t_{3}\right) \ll e_{i} \prod_{j=1}^{3}\left(d_{j}, t_{i}\right) P^{\varepsilon},
$$

so

$$
\begin{aligned}
U\left(N_{1}, N_{2}, N_{3}, L_{1}, L_{2}, L_{3}\right) & \ll \sum_{\mathbf{d}} \sum_{\mathbf{t}} \prod_{i=1}^{3} \prod_{j=1}^{3}\left(d_{j}, t_{i}\right)(1+H) P^{\varepsilon} \\
& \ll\left(D_{1} D_{2} D_{3}\right)^{2}(1+H) P^{\varepsilon} .
\end{aligned}
$$

Now we assume $N_{1} \neq 0$ and $\mathbf{l}=\mathbf{0}$. Let

$$
h=\prod_{\substack{p \mid d_{1} \\ p \nmid d_{2} d_{3} t_{1} t_{2} t_{3}}} p .
$$

The similar argument as before implies $h \mid n_{1}$. So there are at most $4 N_{1} h^{-1}$ choices for $n_{1}$. Then $n_{2}, n_{3}$ will be determined. We arrive at

$$
\begin{aligned}
U\left(N_{1}, N_{2}, N_{3}, L_{1}, L_{2}, L_{3}\right) & \ll \sum_{\mathbf{d}} \sum_{\mathbf{t}}\left(N_{1} h^{-1}\right) d_{1} H N_{1}^{-1} \\
& \ll \sum_{\mathbf{d}} \sum_{\mathbf{t}} \prod_{i}\left(d_{1}, t_{i}\right) H P^{\varepsilon} \\
& \ll\left(D_{1} D_{2} D_{3}\right)^{2} H P^{\varepsilon} .
\end{aligned}
$$

The proof is completed.

To handle the contribution from $\eta \neq 0$, we need the following.

Lemma 13. Let

$$
\mathcal{V}=\sum_{|v| \leq P} \frac{1}{1+|v|} \sum_{\mathbf{d}, \mathbf{t}} \sum_{r \leq P} \frac{1}{r} \sum_{\substack{(\mathbf{n}, \mathbf{l}) \neq \mathbf{0} \in \mathbb{Z}^{6} \\\left|n_{j}\right| \leq d_{j} H P^{\varepsilon},\left|l_{i}\right| \leq t_{i} P^{\varepsilon} \\\left(d_{j}^{2}, r\right)\left|n_{j},\left(t_{i}^{2}, r\right)\right| l_{i} \\ \eta:=\eta\left(\mathbf{d}, \mathbf{t}, \mathbf{n}, \mathbf{l}, v, b_{1}, b_{2}\right) \neq 0}}(r, \eta)(r, \mathbf{d})(r, \mathbf{t}) \max \left\{\frac{n_{j}^{2}}{d_{j}^{2} H^{2}}, \frac{l_{i}^{2}}{t_{i}^{2}}\right\}^{-1}
$$

with $H>0$. One has

$$
\mathcal{V} \ll P^{\varepsilon}\left(D_{1} D_{2} D_{3}\right)^{4}(1+H)^{3}
$$

Proof. By changing variables, we get

$$
\begin{aligned}
\mathcal{V} & \leq \sum_{|v| \leq P} \frac{1}{1+|v|} \sum_{\mathbf{d}, \mathbf{t}} \sum_{\substack{h_{j}\left|d_{j} \\
k_{i}\right| t_{i}}} h_{1} h_{2} h_{3} k_{1} k_{2} k_{3} \sum_{\substack{(\mathbf{n}, \mathbf{l}) \neq \mathbf{0} \in \mathbb{Z}^{6} \\
\left|n_{j}\right| \leq d_{j} H P^{\varepsilon} \\
\left|l_{i}\right| \leq t_{i} P^{\varepsilon} \\
h_{j}\left|n_{j}, k_{i}\right| l_{i} \\
\eta \neq 0}} \max \left\{\frac{n_{j}^{2}}{d_{j}^{2} H^{2}}, \frac{l_{i}^{2}}{t_{i}^{2}}\right\}^{-1} \sum_{r \leq P} \frac{(r, \eta)}{r} \\
& \ll \sum_{\mathbf{d}, \mathbf{t}} \sum_{h_{j} \mid d_{j}} \sum_{k_{i} \mid t_{i}} h_{1} h_{2} h_{3} k_{1} k_{2} k_{3} \sum_{\substack{(\mathbf{n}, \mathbf{l}) \neq \mathbf{0} \in \mathbb{Z}^{6} \\
\left|n_{j}\right| \leq d_{j} H P^{\varepsilon} \\
\left|l_{i}\right| \leq t_{i} P^{\varepsilon} \\
h_{j}\left|n_{j}, k_{i}\right| l_{i}}} \max \left\{\frac{n_{j}^{2}}{d_{j}^{2} H^{2}}, \frac{l_{i}^{2}}{t_{i}^{2}}\right\}^{-1} P^{\varepsilon} .
\end{aligned}
$$


Without loss of generality, we assume

$$
\max \left\{\frac{n_{j}^{2}}{d_{j}^{2} H^{2}}, \frac{l_{i}^{2}}{t_{i}^{2}}\right\}=\frac{n_{1}^{2}}{d_{1}^{2} H^{2}} .
$$

First we handle the case that $\left(n_{2}, n_{3}, l_{1}, l_{2}, l_{3}\right) \in \mathbb{Z}^{5}$ is non-zero. We have

$$
\sum_{h_{j} \mid n_{j}} 1 \leq 1+\left(\left|n_{1}\right| d_{1}^{-1} H^{-1}\right) d_{j} H h_{j}^{-1} \quad \text { for } 2 \leq j \leq 3
$$

and

$$
\sum_{k_{i} \mid l_{i}} 1 \leq 1+\left(\left|n_{1}\right| d_{1}^{-1} H^{-1}\right) t_{i} k_{i}^{-1} \quad \text { for } 1 \leq i \leq 3 .
$$

Since $\left(n_{2}, n_{3}, l_{1}, l_{2}, l_{3}\right) \in \mathbb{Z}^{5}$ is non-zero, at least one of the five inequalities above holds with 1 omitted. Hence

$$
\begin{gathered}
\mathcal{V} \ll \sum_{\mathbf{d}, \mathbf{t}} \sum_{h_{j} \mid d_{j}} \sum_{k_{i} \mid t_{i}} h_{1} h_{2} h_{3} k_{1} k_{2} k_{3} \sum_{\substack{h_{1}\left|n_{1} \\
0<\right| n_{1} \mid \leq d_{1} H P^{\varepsilon}}} \frac{d_{1}^{2} H^{2} P^{\varepsilon}}{n_{1}^{2}}\left(\left(1+\frac{\left|n_{1}\right| d_{2}}{d_{1} h_{2}}\right)\left(1+\frac{\left|n_{1}\right| d_{3}}{d_{1} h_{3}}\right)\right. \\
\left.\times\left(1+\frac{\left|n_{1}\right| t_{1}}{d_{1} H k_{1}}\right)\left(1+\frac{\left|n_{1}\right| t_{2}}{d_{1} H k_{2}}\right)\left(1+\frac{\left|n_{1}\right| t_{3}}{d_{1} H k_{3}}\right)-1\right) .
\end{gathered}
$$

A simple calculation reveals that

$$
\mathcal{V} \ll P^{\varepsilon}\left(D_{1} D_{2} D_{3}\right)^{4}(1+H)^{3} .
$$

Now we turn to the case $n_{2}=n_{3}=l_{1}=l_{2}=l_{3}=0$. Note that $\eta$ is independent of $d_{2}, d_{3}$ and $\mathbf{t}$ in this case. Switching the summations, we arrive at

$$
\begin{aligned}
\mathcal{V} & \ll \sum_{|v| \leq P} \frac{1}{1+|v|} \sum_{d_{1}} \sum_{r \leq P} \frac{1}{r} \sum_{\substack{0<\left|n_{1}\right| \leq d_{1} H P^{\varepsilon} \\
\left(r, d_{1}\right) \mid n_{1} \\
\eta \neq 0}} \frac{d_{1}^{2} H^{2}(r, \eta)\left(r, d_{1}\right)}{n_{1}^{2}} \sum_{d_{2}, d_{3}, \mathbf{t}}\left(r, d_{2}\right)\left(r, d_{3}\right)(r, \mathbf{t}) \\
& \ll \sum_{|v| \leq P} \frac{1}{1+|v|} \sum_{d_{1}} \sum_{r \leq P} \frac{1}{r} \sum_{\substack{0<\left|n_{1}\right| \leq d_{1} H P^{\varepsilon} \\
\eta \neq 0}} d_{1}^{2} H^{2}(r, \eta) n_{1}^{-1} D_{1}\left(D_{2} D_{3}\right)^{2} P^{\varepsilon} .
\end{aligned}
$$

Hence we easily obtain $\mathcal{V} \ll D_{1}^{4} D_{2}^{2} D_{3}^{2} H^{2} P^{\varepsilon}$. The proof is thus completed.

For $H>0$, we define

$$
\mathcal{N}_{\mathbf{d}, q}(H)=\left\{\mathbf{n} \in \mathbb{Z}^{3}:\left|n_{i}\right| \leq d_{i} H P^{\varepsilon}, n_{i} \equiv 0\left(\bmod \left(q, d_{i}^{2}\right)\right), i=1,2,3\right\},
$$

and write $\mathcal{N}_{\mathbf{d}, q}=\mathcal{N}_{\mathbf{d}, q}(1)$. Let

$$
\begin{aligned}
y_{1}=\sum_{|v| \leq P} & \frac{1}{1+|v|} \sum_{\substack{\mathbf{d}, \mathbf{t} \\
\Delta \mid}} \sum_{\Delta \mid\left(p p^{\prime}\right)^{2}} \sum_{q \leq P} \frac{\Delta^{3}}{q^{6}} \\
& \times \sum_{\substack{\mathbf{n} \in \mathcal{N}_{q, \mathbf{d}}\left(p p^{\prime} / \Delta\right) \\
\mathbf{l} \in \mathcal{N}_{q, \mathbf{t}}}}\left|W\left(\Delta, q ; \mathbf{d}, \mathbf{t}, \mathbf{n}, \mathbf{l}, p, p^{\prime}, v\right)\right| \mathcal{C}\left(q, \mathbf{d}, \mathbf{t}, \mathbf{n}, \mathbf{l}, p p^{\prime} \Delta^{-1}\right),
\end{aligned}
$$


where

$$
\digamma(q, \mathbf{d}, \mathbf{t}, \mathbf{n}, \mathbf{l}, H)= \begin{cases}\min & \text { if }(\mathbf{n}, \mathbf{l}) \neq \mathbf{0} \\ q^{2} P^{-2} & \text { if }(\mathbf{n}, \mathbf{l})=\mathbf{0}\end{cases}
$$

and

$$
\min =\min \left\{\frac{q^{2}}{P^{2}}\left(\sum_{j=1}^{3}\left(\frac{n_{j}^{2}}{d_{j}^{2} H^{2}}+\frac{l_{i}^{2}}{t_{i}^{2}}\right)\right)^{-1}, \frac{q}{P}\left(\sum_{j=1}^{3}\left(\frac{\left|n_{j}\right|}{d_{j} H}+\frac{\left|l_{i}\right|}{t_{i}}\right)\right)^{-1}\right\}
$$

Let

$$
\begin{aligned}
y_{2}=\sum_{|v| \leq P} & \frac{1}{1+|v|} \sum_{\mathbf{d}} \sum_{\mathbf{t}} \sum_{q \leq P} \frac{1}{q^{6}} \sum_{\substack{(\mathbf{n}, \mathbf{l}) \neq \mathbf{0} \in \mathbb{Z}^{6} \\
\mathbf{n} \in \mathcal{N}_{q, \mathbf{d}} \\
\mathbf{1} \in \mathcal{N}_{q, \mathbf{t}}}}|\theta(q ; \mathbf{d}, \mathbf{t}, \mathbf{n}, \mathbf{l}, v, 1,1)| \\
& \times \min \left\{\frac{q^{2}}{P^{2}}\left(\sum_{j=1}^{3}\left(\frac{n_{j}^{2}}{d_{j}^{2}}+\frac{l_{i}^{2}}{t_{i}^{2}}\right)\right)^{-1}, \frac{q}{P}\left(\sum_{j=1}^{3}\left(\frac{\left|n_{j}\right|}{d_{j}}+\frac{\left|l_{i}\right|}{t_{i}}\right)\right)^{-1}\right\} .
\end{aligned}
$$

With the help of Lemmas 8-13, we establish the following result.

Lemma 14. Let $y_{1}$ and $y_{2}$ be defined as above. Then one has

$$
\begin{aligned}
& y_{1} \ll\left(D_{1} D_{2} D_{3}\right)^{2} P^{\varepsilon-1} R^{6}+\left(D_{1} D_{2} D_{3}\right)^{4} P^{-2+\varepsilon} R^{10}, \\
& y_{2} \ll\left(D_{1} D_{2} D_{3}\right)^{2} P^{-1+\varepsilon}+\left(D_{1} D_{2} D_{3}\right)^{4} P^{-2+\varepsilon} .
\end{aligned}
$$

Proof. The proofs for $y_{1}$ and $y_{2}$ are similar. Indeed, the argument for $y_{2}$ is easier, because there is no $R$ involved. We only work out the details for $y_{1}$.

We write $y_{1}=y_{1}^{(1)}+y_{1}^{(2)}+y_{1}^{(3)}$, where $y_{1}^{(1)}$ is the contribution from $(\mathbf{n}, \mathbf{l})=\mathbf{0}, y_{1}^{(2)}$ is the contribution from those terms with $(\mathbf{n}, \mathbf{l}) \neq \mathbf{0}$ but $\eta:=\eta\left(\mathbf{d}, \mathbf{t}, \mathbf{n}, \mathbf{l}, v, \pi \pi^{\prime}, \pi \pi^{\prime} p p^{\prime} / \Delta\right)=0$, and $y_{1}^{(3)}$ is the contribution from the remaining terms. By Lemma 11 , when $(\mathbf{n}, \mathbf{l})=\mathbf{0}$ or $\eta=0$,

$$
\begin{aligned}
& W\left(\Delta, q ; \mathbf{d}, \mathbf{t}, \mathbf{n}, \mathbf{l}, p, p^{\prime}, v\right) \\
& \quad \ll 2^{4 u} r^{4}\left(r, \mathbf{d}^{2}\right)^{1 / 2}\left(r, \mathbf{t}^{2}\right)^{1 / 2} \Delta^{-2} R^{4}\left(\pi \pi^{\prime}\right)^{5}\left(1+\Delta R^{-2}\right)^{-1},
\end{aligned}
$$

where we have used the decomposition $q=2^{u} \pi \pi^{\prime} r$ with $\left(2 p p^{\prime}, r\right)=1$. On applying the estimate (4.7), we get

$$
y_{1}^{(1)} \ll P^{-2} R^{4} \sum_{|v| \leq P} \frac{1}{1+|v|} \sum_{\mathbf{d}, \mathbf{t}} \sum_{\Delta \mid\left(p p^{\prime}\right)^{2}} \sum_{2^{u} \pi \pi^{\prime} r \leq P} \frac{\Delta \pi \pi^{\prime}\left(r, \mathbf{d}^{2}\right)^{1 / 2}\left(r, \mathbf{t}^{2}\right)^{1 / 2}}{1+\Delta R^{-2}} .
$$

Recalling the condition (2.8), we obtain

$$
\begin{aligned}
\sum_{r \leq \frac{P}{2^{u} \pi \pi^{\prime}}}\left(r, \mathbf{d}^{2}\right)^{1 / 2}\left(r, \mathbf{t}^{2}\right)^{1 / 2} & \leq\left(\sum_{r \leq \frac{P}{2^{u} \pi \pi^{\prime}}}\left(r, \mathbf{d}^{2}\right)\right)^{1 / 2}\left(\sum_{r \leq \frac{P}{2^{u} \pi \pi^{\prime}}}\left(r, \mathbf{t}^{2}\right)\right)^{1 / 2} \\
& \ll P^{\varepsilon}\left(\sum_{r \leq \frac{P}{2^{u} \pi \pi^{\prime}}}\left(r, d_{1}^{2} d_{2}^{2} d_{3}^{2}\right)\right)^{1 / 2}\left(\sum_{r \leq \frac{P}{2^{u} \pi \pi^{\prime}}}\left(r, t_{1}^{2} t_{2}^{2} t_{3}^{2}\right)\right)^{1 / 2} \\
& \ll \frac{P^{1+\varepsilon}}{2^{u} \pi \pi^{\prime}} .
\end{aligned}
$$


Then one can get

$$
\sum_{2^{u} \pi \pi^{\prime} r \leq P} \pi \pi^{\prime}\left(r, \mathbf{d}^{2}\right)^{1 / 2}\left(r, \mathbf{t}^{2}\right)^{1 / 2} \ll P^{1+\varepsilon} .
$$

It is easy to see

$$
\sum_{\Delta \mid\left(p p^{\prime}\right)^{2}} \frac{\Delta}{1+\Delta R^{-2}} \ll R^{2}
$$

We can now conclude that

$$
y_{1}^{(1)} \ll\left(D_{1} D_{2} D_{3}\right)^{2} P^{\varepsilon-1} R^{6} .
$$

Combining (4.7) and

$$
e\left(q, \mathbf{d}, \mathbf{t}, \mathbf{n}, \mathbf{l}, \frac{p p^{\prime}}{\Delta}\right) \ll \frac{q}{P} \max \left\{\frac{\left|n_{j}\right|}{d_{j} p p^{\prime} / \Delta}, \frac{\left|l_{i}\right|}{t_{i}}\right\}^{-1},
$$

one can deduce that

$$
\begin{gathered}
y_{1}^{(2)} \ll P^{-1} R^{4} \sum_{|v| \leq P} \frac{1}{1+|v|} \sum_{\mathbf{d}, \mathbf{t}} \sum_{\Delta \mid\left(p p^{\prime}\right)^{2}} \sum_{2^{u} \pi \pi^{\prime} r \leq P} \sum_{\substack{(\mathbf{n}, \mathbf{l}) \neq \mathbf{0} \in \mathbb{Z}^{6} \\
\left|n_{j}\right| \leq d_{j} P^{\varepsilon} p p^{\prime} / \Delta \\
\left|l_{i}\right| \leq t_{i} P^{\varepsilon} \\
\eta=0}} \frac{\Delta}{2^{u} r\left(1+\Delta R^{-2}\right)} \\
\times\left(r, \mathbf{d}^{2}\right)^{1 / 2}\left(r, \mathbf{t}^{2}\right)^{1 / 2} \max \left\{\frac{\left|n_{j}\right|}{d_{j} p p^{\prime} / \Delta}, \frac{\left|l_{i}\right|}{t_{i}}\right\}^{-1} .
\end{gathered}
$$

Note that

$$
\sum_{2^{u} \pi \pi^{\prime} r \leq P} \frac{1}{2^{u} r}\left(r, \mathbf{d}^{2}\right)^{1 / 2}\left(r, \mathbf{t}^{2}\right)^{1 / 2} \ll P^{\varepsilon},
$$

we have

$$
\begin{gathered}
y_{1}^{(2)} \ll P^{-1+\varepsilon} R^{4} \sum_{\Delta \mid\left(p p^{\prime}\right)^{2}} \frac{\Delta}{1+\Delta R^{-2}} \sum_{|v| \leq P} \frac{1}{1+|v|} \\
\times \sum_{\mathbf{d}, \mathbf{t}} \sum_{\substack{(\mathbf{n}, \mathbf{l}) \neq \mathbf{l} \neq \mathbf{0} \in \mathbb{Z}^{6} \\
\left|n_{j}\right| \leq d_{j} P^{\varepsilon} p p^{\prime} / \Delta \\
\left|l_{i}\right| \leq t_{i} P^{\varepsilon} \\
\eta=0}} \max \left\{\frac{\left|n_{j}\right|}{d_{j} p p^{\prime} / \Delta}, \frac{\left|l_{i}\right|}{t_{i}}\right\}^{-1} .
\end{gathered}
$$

Then one can conclude, by appealing to Lemma 12, that

$$
\begin{aligned}
y_{1}^{(2)} & \ll P^{-1+\varepsilon} R^{4} \sum_{\Delta \mid\left(p p^{\prime}\right)^{2}} \frac{\Delta}{1+\Delta R^{-2}}\left(D_{1} D_{2} D_{3}\right)^{2}\left(1+\frac{p p^{\prime}}{\Delta}\right) \\
& \ll\left(D_{1} D_{2} D_{3}\right)^{2} P^{\varepsilon-1} R^{6} .
\end{aligned}
$$

For $y_{1}^{(3)}$ we shall apply

$$
W\left(\Delta, q ; \mathbf{d}, \mathbf{t}, \mathbf{n}, \mathbf{l}, p, p^{\prime}, v\right) \ll 2^{4 u} r^{3}(r, \eta)(r, \mathbf{d})(r, \mathbf{t}) R^{4}\left(\pi \pi^{\prime}\right)^{4}
$$

by Lemma 11 . Note that $\mathcal{N}_{q, \mathbf{d}}\left(p p^{\prime} / \Delta\right)=\mathcal{N}_{r, \mathbf{d}}\left(p p^{\prime} / \Delta\right)$ for $q=2^{u} \pi \pi^{\prime} r$, and

$$
\varphi\left(q, \mathbf{d}, \mathbf{t}, \mathbf{n}, \mathbf{l}, \frac{p p^{\prime}}{\Delta}\right) \ll \frac{q^{2}}{P^{2}} \max \left\{\frac{n_{j}^{2}}{d_{j}^{2}\left(p p^{\prime} / \Delta\right)^{2}}, \frac{l_{i}^{2}}{t_{i}^{2}}\right\}^{-1} \text {. }
$$


Thus, one has

$$
\begin{aligned}
y_{1}^{(3)} \ll P^{-2} R^{4} & \sum_{\Delta \mid\left(p p^{\prime}\right)^{2}} \Delta \sum_{|v| \leq P} \frac{1}{1+|v|} \sum_{2^{u} \pi \pi^{\prime} r \leq P} \frac{1}{r} \\
\times & \sum_{\mathbf{d}, \mathbf{t}} \sum_{\substack{(\mathbf{n}, \mathbf{l}) \neq \mathbf{0} \in \mathbb{Z}^{6} \\
\mathbf{n} \in \mathcal{N}_{r, \mathbf{d}}\left(\Delta /\left(p p^{\prime}\right)\right) \\
\mathbf{i} \in \mathcal{N}_{r, \mathbf{t}}}}(r, \eta)(r, \mathbf{d})(r, \mathbf{t}) \max \left\{\frac{n_{j}^{2}}{d_{j}^{2}\left(p p^{\prime} / \Delta\right)^{2}}, \frac{l_{i}^{2}}{t_{i}^{2}}\right\}^{-1} .
\end{aligned}
$$

By Lemma 13, we have

$$
y_{1}^{(3)} \ll P^{-2+\varepsilon} R^{4} \sum_{\Delta \mid\left(p p^{\prime}\right)^{2}} \Delta\left(D_{1} D_{2} D_{3}\right)^{4}\left(1+\frac{p p^{\prime}}{\Delta}\right)^{3} \ll\left(D_{1} D_{2} D_{3}\right)^{4} P^{\varepsilon-2} R^{10} .
$$

The assertion is established.

Lemma 15. Let

$$
\begin{aligned}
\mathcal{F}= & \sum_{0<|l| \leq} R^{2} Q^{2} P^{\varepsilon-2} \\
& \times \sum_{\substack{q_{1}, q_{2} \\
\left(q_{1} q_{2}, p p^{\prime}\right)=1 \\
|l|\left(p p^{\prime}\right)^{-1} P^{2-\varepsilon}<q_{1} q_{2}}}\left(\frac{l}{p p^{\prime}}\right) \\
& \times \sum_{\substack{a_{1}\left(q_{1}\right)^{*}, a_{2}\left(q_{2}\right)^{*} \\
a^{\prime}\left(a_{1} q_{2}+a_{2} q_{1}\right) \equiv l\left(\bmod q_{1} q_{2}\right)}}^{-3}\left(\frac{q_{1} q_{2}}{p p^{\prime}}\right) e_{p p^{\prime}}\left(-\overline{q_{1} q_{2}} l N\right) e\left(\frac{l n}{q_{1} q_{2} p p^{\prime}}\right)
\end{aligned}
$$

Then we have

$$
\mathcal{F} \ll P^{-4+\varepsilon} Q^{3} R^{2}+P^{-4+\varepsilon} Q^{14 / 5} R^{24 / 5} .
$$

Proof. We modify the argument of Heath-Brown and Tolev [9]. Let

$$
q_{j}=g_{j} b_{j}, \quad 1 \leq j \leq 2,
$$

where

$$
g_{1}=\prod_{\substack{p^{k} \| q_{1} \\ p \nmid 2 d_{1} d_{2} d_{3}\left(q_{1}, q_{2}\right)}} p^{k} \text { and } g_{2}=\prod_{\substack{p^{k} \| q_{2} \\ p \nmid 2 t_{1} t_{2} t_{3}\left(q_{1}, q_{2}\right)}} p^{k}
$$

Then

$$
\left(g_{1}, g_{2}\right)=\left(g_{1} g_{2}, b_{1} b_{2}\right)=\left(g_{1}, 2 d_{1} d_{2} d_{3}\right)=\left(g_{2}, 2 t_{1} t_{2} t_{3}\right)=1 .
$$

Let $a_{j}=\alpha_{j} b_{j}+\beta_{j} g_{j}$ for $j=1,2$. Then

$$
p p^{\prime}\left(a_{1} q_{2}+a_{2} q_{1}\right) \equiv l\left(\bmod q_{1} q_{2}\right)
$$

is equivalent to the three conditions

$$
\begin{aligned}
p p^{\prime} \alpha_{1} b_{1} b_{2} g_{2} & \equiv l\left(\bmod g_{1}\right), \\
p p^{\prime} \alpha_{2} b_{1} b_{2} g_{1} & \equiv l\left(\bmod g_{2}\right), \\
p p^{\prime} g_{1} g_{2}\left(\beta_{1} b_{2}+\beta_{2} b_{1}\right) & \equiv l\left(\bmod b_{1} b_{2}\right) .
\end{aligned}
$$


Hence by Lemma 1 (i), the inner multiple sum over $a_{1}, a_{2}$ in $\mathcal{F}$ is equal to

$$
\left(\frac{-p p^{\prime} b_{1} b_{2} g_{2} l}{g_{1}}\right)\left(\frac{-p p^{\prime} b_{1} b_{2} g_{1} l}{g_{2}}\right) S^{3}\left(g_{1}, 1\right) S^{3}\left(g_{2}, 1\right) \Xi
$$

where

$$
\Xi=\Xi\left(p p^{\prime} g_{1} g_{2}\right)=\sum_{\substack{\beta_{1}\left(b_{1}\right)^{*}, \beta_{2}\left(b_{2}\right)^{*} \\ p p^{\prime} g_{1} g_{2}\left(\beta_{1} b_{2}+\beta_{2} b_{1}\right) \equiv l\left(\bmod b_{1} b_{2}\right)}} S_{\mathbf{d}}\left(b_{1},-\beta_{1}\right) S_{\mathbf{t}}\left(b_{2},-\beta_{2}\right) .
$$

Let $b_{j}=B_{j} \Delta$ for $j=1,2$ with $\Delta=\left(b_{1}, b_{2}\right)$. Then $\Xi=0$ if $\Delta \nmid l$, and for $l=\Delta v$ we have

$$
\Xi\left(p p^{\prime} g_{1} g_{2}\right)=\sum_{\substack{\beta_{1}\left(B_{1} \Delta\right)^{*}, \beta_{2}\left(B_{2} \Delta\right)^{*} \\ p p^{\prime} g_{1} g_{2}\left(\beta_{1} B_{2}+\beta_{2} B_{1}\right) \equiv v\left(\bmod B_{1} B_{2} \Delta\right)}} S_{\mathbf{d}}\left(B_{1} \Delta, \beta_{1}\right) S_{\mathbf{t}}\left(B_{2} \Delta, \beta_{2}\right) .
$$

In order to change variables, we introduce the conditions

$$
\left\{\begin{aligned}
\left(g_{1}, g_{2}\right)=\left(g_{1} g_{2}, B_{1} B_{2} \Delta\right)=\left(B_{1}, B_{2}\right) & =1, \\
\left(g_{1}, 2 d_{1} d_{2} d_{3}\right)=\left(g_{2}, 2 t_{1} t_{2} t_{3}\right) & =1, \\
\rho_{\mathbf{d}}\left(B_{1} \Delta\right)=\rho_{\mathbf{d}}(\Delta), \quad \rho_{\mathbf{t}}\left(B_{2} \Delta\right) & =\rho_{\mathbf{t}}(\Delta), \\
\left(B_{1} B_{2} \Delta g_{1} g_{2}, p p^{\prime}\right)=1, \quad|v|\left(p p^{\prime}\right)^{-1} P^{2-\varepsilon} & <B_{1} B_{2} \Delta g_{1} g_{2},
\end{aligned}\right.
$$

where for $\mathbf{k}=\left(k_{1}, k_{2}, k_{3}\right)$ we use the notation

$$
\rho_{\mathbf{k}}(m)=\prod_{\substack{p \mid m \\ p \nmid 2 k_{1} k_{2} k_{3}}} p .
$$

By changing variables we arrive at

$$
\begin{aligned}
& \mathcal{F}= \sum_{\substack{v, \Delta \\
0<|v \Delta| \leq R^{2} Q^{2} P^{\varepsilon-2}}}\left(\frac{v \Delta}{p p^{\prime}}\right) \sum_{B_{1}, B_{2}}\left(B_{1} B_{2}\right)^{-3} \Delta^{-6}\left(\frac{B_{1} B_{2}}{p p^{\prime}}\right) \\
& \times \sum_{\substack{g_{1}, g_{2} \\
(4.8)}}\left(g_{1} g_{2}\right)^{-3}\left(\frac{g_{1} g_{2}}{p p^{\prime}}\right) e_{p p^{\prime}}\left(-\overline{\left.g_{1} g_{2} B_{1} B_{2} \Delta v N\right) e\left(\frac{v n}{g_{1} g_{2} B_{1} B_{2} \Delta p p^{\prime}}\right)}\right. \\
& \quad \times\left(\frac{-p p^{\prime} B_{1} B_{2} g_{2} \Delta v}{g_{1}}\right)\left(\frac{-p p^{\prime} B_{1} B_{2} g_{1} \Delta v}{g_{2}}\right) S^{3}\left(g_{1}, 1\right) S^{3}\left(g_{2}, 1\right) \Xi\left(p p^{\prime} g_{1} g_{2}\right) .
\end{aligned}
$$

Let

$$
\xi\left(g_{1}, g_{2}\right)=e\left(\frac{v n}{g_{1} g_{2} B_{1} B_{2} \Delta p p^{\prime}}\right)\left(\frac{g_{1} g_{2}}{p p^{\prime}}\right)\left(\frac{-p p^{\prime} g_{2}}{g_{1}}\right)\left(\frac{-p p^{\prime} g_{1}}{g_{2}}\right) \frac{S^{3}\left(g_{1}, 1\right) S^{3}\left(g_{2}, 1\right)}{g_{1}^{3} g_{2}^{3}} .
$$

Note that $\Xi(\lambda)$ depends on $\lambda\left(\bmod B_{1} B_{2} \Delta\right)$ only. Hence

$$
\begin{aligned}
& \mathcal{F}= \sum_{\substack{v, \Delta \\
\left(v \Delta, p p^{\prime}\right)=1 \\
0<|v \Delta| \leq R^{2} Q^{2} P^{\varepsilon-2}}}\left(\frac{v}{p p^{\prime}}\right) \sum_{B_{1}, B_{2}}\left(B_{1} B_{2}\right)^{-3} \Delta^{-6} \sum_{\lambda\left(\bmod 8 B_{1} B_{2} \Delta\right)} \Xi(\lambda)\left(\frac{B_{1} B_{2} \Delta}{\lambda}\right) \\
& \times \sum_{\substack{g_{1}, g_{2} \\
(4.8) \\
p p^{\prime} g_{1} g_{2} \equiv \lambda\left(\bmod 8 B_{1} B_{2} \Delta\right)}} e_{p p^{\prime}}\left(-\overline{g_{1} g_{2} B_{1} B_{2} \Delta} v N\right)\left(\frac{v}{g_{1}}\right)\left(\frac{v}{g_{2}}\right) \xi\left(g_{1}, g_{2}\right) .
\end{aligned}
$$


Moreover, in view of (4.8) and the condition

$$
p p^{\prime} g_{1} g_{2} \equiv \lambda\left(\bmod 8 B_{1} B_{2} \Delta\right),
$$

the summation $\sum_{\lambda\left(\bmod 8 B_{1} B_{2} \Delta\right)}$ can be replaced by $\sum_{\lambda}\left(\bmod 8 B_{1} B_{2} \Delta\right)^{*}$.

From Lemma 1 (ii), (iii), (iv), and (2.8),

$$
\Xi(\lambda) \ll \Delta^{4}\left(B_{1} B_{2}\right)^{3 / 2}\left(\Delta B_{1}, \mathbf{d}^{2}\right)^{1 / 2}\left(\Delta B_{2}, \mathbf{t}^{2}\right)^{1 / 2} \ll \Delta^{5}\left(B_{1} B_{2}\right)^{2} P^{\varepsilon} .
$$

By the dyadic argument, we have

$$
\mathcal{F} \ll \log ^{2} P \sup _{\substack{G_{1} \leq G_{1}^{\prime} \leq 2 G_{1} \\ G_{2} \leq G_{2}^{\prime} \leq 2 G_{2}}} \mathcal{F}\left(G_{1}, G_{2}\right),
$$

where

$$
\begin{aligned}
& \mathcal{F}\left(G_{1}, G_{2}\right)= \sum_{\substack{v, \Delta \\
\left(v \Delta, p p^{\prime}\right)=1 \\
0<|v \Delta| \leq R^{2} Q^{2} P^{\varepsilon-2}}} \sum_{B_{1}, B_{2}}\left(B_{1} B_{2}\right)^{-1} \Delta^{-1} \sum_{\lambda\left(\bmod 8 B_{1} B_{2} \Delta\right)^{*}} \\
& \times \sum_{\substack{G_{1} \leq g_{1} \leq G_{1}^{\prime}, G_{2} \leq g_{2} \leq G_{2}^{\prime} \\
(4.8)}} e_{p p^{\prime}}\left(-\overline{g_{1} g_{2} B_{1} B_{2} \Delta} v N\right)\left(\frac{v}{g_{1}}\right)\left(\frac{v}{g_{2}}\right) \xi\left(g_{1}, g_{2}\right) \mid . \\
& p p^{\prime} g_{1} g_{2} \equiv \lambda\left(\bmod 8 B_{1} B_{2} \Delta\right)
\end{aligned}
$$

Without loss of generality, we assume $G_{1} \leq G_{2}$. In view of (4.8), the multiple summations over $B_{1}, B_{2}$ are naturally restricted by

$$
\left\{\begin{aligned}
\rho_{\mathbf{d}}\left(B_{1} \Delta\right) & =\rho_{\mathbf{d}}(\Delta), \quad \rho_{\mathbf{t}}\left(B_{2} \Delta\right)=\rho_{\mathbf{t}}(\Delta), \\
\left(B_{1} B_{2}, p p^{\prime}\right) & =1, \\
|v| R^{-2} P^{2-\varepsilon} & \leq 16 B_{1} B_{2} \Delta G_{1} G_{2} .
\end{aligned}\right.
$$

In view of the congruence condition $p p^{\prime} g_{1} g_{2} \equiv \lambda\left(\bmod 8 B_{1} B_{2} \Delta\right)$, for fixed $p, p^{\prime}, \lambda$ and $g_{1}$, we have

$$
\xi\left(g_{1}, g_{2}\right)=e\left(\frac{v n}{g_{1} g_{2} B_{1} B_{2} \Delta p p^{\prime}}\right) g_{1}^{-3 / 2} g_{2}^{-3 / 2} \epsilon,
$$

where $|\epsilon|=1$ and $\epsilon$ is independent of $g_{2}$. Partial summation gives

$$
\begin{gathered}
\mathcal{F}\left(G_{1}, G_{2}\right) \ll \sup _{\substack{G_{2} \leq G, G^{\prime} \leq G_{2}^{\prime} \\
G_{1} \leq g_{1} \leq G_{1}^{\prime}}} G_{1}^{-1 / 2} G_{2}^{-3 / 2} P^{\varepsilon} \sum_{\substack{v, \Delta \\
\left(v \Delta, p p^{\prime}\right)=1 \\
0<|v \Delta| \leq R^{2} Q^{2} P^{\varepsilon-2}}} \Delta^{-1} \sum_{\substack{B_{1}, B_{2} \\
(4.10)}}\left(B_{1} B_{2}\right)^{-1} \\
\times \sum_{\lambda\left(\bmod 8 B_{1} B_{2} \Delta\right)^{*}} \sum_{\substack{G \leq g_{2} \leq G^{\prime} \\
(4.11) \\
p p^{\prime} g_{1} g_{2} \equiv \lambda\left(\bmod 8 B_{1} B_{2} \Delta\right)}} e_{p p^{\prime}}\left(-\overline{g_{1} g_{2} B_{1} B_{2} \Delta} v N\right)\left(\frac{v}{g_{2}}\right) \mid,
\end{gathered}
$$

where the condition (4.11) comprises

$$
\left\{\begin{aligned}
\left(g_{1}, g_{2}\right)=\left(g_{2}, B_{1} B_{2} \Delta\right) & =1, \\
\left(g_{2}, 2 t_{1} t_{2} t_{3}\right) & =1, \\
\left(g_{2}, p p^{\prime}\right)=1, \quad|v|\left(p p^{\prime}\right)^{-1} P^{2-\varepsilon} & <B_{1} B_{2} \Delta g_{1} g_{2} .
\end{aligned}\right.
$$


As in [9], we use two different estimates according to $G_{2} \geq H$ or otherwise, where $H$ will be chosen later. The summation over $g_{2}$ is of the type

$$
\mathcal{M}=\sum_{\substack{K \leq g \leq K^{\prime} \\(g, u)=1 \\ g \equiv \lambda^{\prime}\left(\bmod 8 B_{1} B_{2} \Delta\right)}} e_{p p^{\prime}}\left(\overline{g m_{1}} m_{2}\right)\left(\frac{v}{g}\right),
$$

where $G \leq K, K^{\prime} \leq G^{\prime}$ and $\left(m_{1} m_{2}, p p^{\prime}\right)=\left(2 B_{1} B_{2} \Delta, \lambda^{\prime}\right)=1$. We shall prove

$$
\mathcal{M} \ll P^{\varepsilon} G_{2}\left(B_{1} B_{2} \Delta p p^{\prime}\right)^{-1}+|v| R^{2} P^{\varepsilon} .
$$

With (4.13), we can get

$$
\begin{aligned}
& \mathcal{F}\left(G_{1}, G_{2}\right) \ll G_{1}^{-1 / 2} G_{2}^{-3 / 2} P^{\varepsilon} \quad \sum_{v, \Delta} \sum_{B_{1}, B_{2}}\left(G_{2}\left(B_{1} B_{2} \Delta p p^{\prime}\right)^{-1}+|v| R^{2}\right) \\
& 0<|v \Delta| \leq R^{2} Q^{2} P^{\varepsilon-2}(4.10) \\
& \ll G_{1}^{-1 / 2} G_{2}^{-3 / 2} P^{\varepsilon} \sum_{\substack{v, \Delta \\
0<|v \Delta| \leq R^{2} Q^{2} P^{\varepsilon-2}}}\left(|v|^{-1} P^{-2} G_{1} G_{2}^{2}+|v| R^{2}\right) \sum_{\substack{B_{1}, B_{2} \\
(4.10)}} 1 .
\end{aligned}
$$

Note that

$$
\sum_{\substack{B_{1}, B_{2} \\(4.10)}} 1 \leq \sum_{\substack{B_{1} \leq P \\ p\left|B_{1} \Rightarrow p\right| 2 d_{1} d_{2} d_{3} \Delta}} \sum_{\substack{B_{2} \leq P \\ B_{2} \Rightarrow p \mid 2 t_{1} t_{2} t_{3} \Delta}} 1 \ll P^{\varepsilon}
$$

We now conclude

$$
\begin{aligned}
\mathcal{F}\left(G_{1}, G_{2}\right) & \ll P^{-4+\varepsilon} R^{2} Q^{2} G_{1}^{1 / 2} G_{2}^{1 / 2}+G_{1}^{-1 / 2} G_{2}^{-3 / 2} R^{6} Q^{4} P^{-4+\varepsilon} \\
& \ll P^{-4+\varepsilon} R^{2} Q^{3}+R^{6} Q^{4} P^{-4+\varepsilon} H^{-3 / 2} .
\end{aligned}
$$

To prove (4.13), we first remove the restriction $(g, u)=1$, getting

$$
\mathcal{M}=\sum_{w \mid u} \mu(w)\left(\frac{v}{w}\right) \sum_{\substack{K w^{-1} \leq g \leq K^{\prime} w^{-1} \\ g w \equiv \lambda^{\prime}\left(\bmod 8 B_{1} B_{2} \Delta\right)}} e_{p p^{\prime}}\left(\overline{g w m_{1}} m_{2}\right)\left(\frac{v}{g}\right) .
$$

We divide the inner summation into $O\left(G_{2} w^{-1}\left(8 B_{1} B_{2} \Delta p p^{\prime}|v|\right)^{-1}\right)$ complete sums and at most one incomplete sum

$$
\mathcal{M} \ll \sum_{w \mid u}\left(G_{2} w^{-1}\left(8 B_{1} B_{2} \Delta p p^{\prime}|v|\right)^{-1}\left|\mathcal{M}_{0}\right|+O\left(|v| R^{2}\right)\right),
$$

where

$$
\mathcal{M}_{0}=\sum_{\substack{g\left(\bmod 8 B_{1} B_{2} \Delta p p^{\prime}|v|\right) \\ g w \equiv \lambda^{\prime}\left(\bmod 8 B_{1} B_{2} \Delta\right)}} e_{p p^{\prime}}\left(\overline{g w m_{1}} m_{2}\right)\left(\frac{v}{g}\right)
$$

and the error $O\left(|v| R^{2}\right)$ is the contribution from the incomplete sum. Recalling the condition $\left(B_{1} B_{2} \Delta v, p p^{\prime}\right)=1$, we change variables, by $g=s 8 B_{1} B_{2} \Delta|v|+k p p^{\prime}$, to deduce that

$$
\mathcal{M}_{0}=\sum_{\substack{k\left(\bmod 8 B_{1} B_{2} \Delta|v|\right) \\ k p p^{\prime} w \equiv \lambda^{\prime}\left(\bmod 8 B_{1} B_{2} \Delta\right)}}\left(\frac{v}{k p p^{\prime}}\right) \sum_{s\left(\bmod p p^{\prime}\right)^{*}} e_{p p^{\prime}}\left(\overline{s 8 B_{1} B_{2} \Delta|v| w m_{1}} m_{2}\right) .
$$

The inner sum is a Ramanujan sum, so $\mathcal{M}_{0} \ll|v|$. Now (4.13) follows from (4.15). 
When $G_{2}<H$, we apply the trivial bound to get

$$
\begin{aligned}
\mathscr{F}\left(G_{1}, G_{2}\right) \ll \sup _{G, G^{\prime}} G_{1}^{-1 / 2} G_{2}^{-3 / 2} \sum_{\substack{v, \Delta \\
0<|v \Delta| \leq R^{2} Q^{2} P^{\varepsilon-2}}} \Delta^{-1} \\
\times \sum_{B_{1}, B_{2}}\left(|v| R^{-2} P^{2-\varepsilon}\left(\Delta G_{1} G_{2}\right)^{-1}\right)^{-1} G_{2} .
\end{aligned}
$$

Recalling (4.14), we arrive at

$$
\mathcal{F}\left(G_{1}, G_{2}\right) \ll P^{-4+\varepsilon} R^{4} Q^{2} G_{1}^{1 / 2} G_{2}^{1 / 2} \ll P^{-4+\varepsilon} R^{4} Q^{2} H
$$

We choose

$$
H=R^{4 / 5} Q^{4 / 5},
$$

by equating $R^{6} Q^{4} P^{-4} H^{-3 / 2}=P^{-4} R^{4} Q^{2} H$, to conclude finally

$$
\mathcal{F}\left(G_{1}, G_{2}\right) \ll P^{-4+\varepsilon} Q^{3} R^{2}+P^{-4+\varepsilon} Q^{14 / 5} R^{24 / 5} .
$$

The proof is completed by putting (4.17) into (4.9).

\section{Invoking the square sieve}

By the dyadic argument, our task is to prove

$$
\sum_{k \leq P}\left|\sum_{D_{i}^{\prime} \leq d_{i}<2 D_{i}^{\prime}(1 \leq i \leq 3)} \beta(\mathbf{d})\left(\Omega_{\mathbf{d}}\left(N-k^{2}\right)-\mathcal{M}_{\mathbf{d}, Q}\left(N-k^{2}\right)\right)\right| \ll P^{2-\varepsilon} .
$$

Suppose that $\kappa \in(0,1 / 2)$. Set $m=4\left\lceil\kappa^{-1}\right\rceil$, and denote by $\mathcal{R}_{j}$ the interval $\left[2^{j-1} P^{\kappa}, 2^{j} P^{\kappa}\right)$ for $1 \leq j \leq m$. Let $\pi(\mathcal{R})=\prod_{p \in \mathcal{R}} p$. We have the partitions

$$
\left[D_{i}^{\prime}, 2 D_{i}^{\prime}\right)=\bigsqcup_{J \subseteq\{1, \ldots, m\}} \mathscr{D}_{J},
$$

where $\mathscr{D}_{J}=\left\{D_{i}^{\prime} \leq d_{i}<2 D_{i}^{\prime}:\left(d_{i}, \pi\left(\mathcal{R}_{j}\right)\right) \neq 1 \Longleftrightarrow j \in J\right\}$. Hence (5.1) can be deduced from

$$
\sum_{k \leq P}\left|\sum_{d_{i} \in D_{J_{i}}(1 \leq i \leq 3)} \beta(\mathbf{d})\left(\Omega_{\mathbf{d}}\left(N-k^{2}\right)-\mathcal{M}_{\mathbf{d}, Q}\left(N-k^{2}\right)\right)\right| \ll P^{2-\varepsilon} .
$$

By Cauchy's inequality, it suffices to prove

$$
\sum_{k \leq P}\left|\sum_{d_{i} \in D_{J_{i}}(1 \leq i \leq 3)} \beta(\mathbf{d})\left(\Omega_{\mathbf{d}}\left(N-k^{2}\right)-\mathcal{M}_{\mathbf{d}, Q}\left(N-k^{2}\right)\right)\right|^{2} \ll P^{3-\varepsilon} .
$$

Note that $D_{i}^{\prime} \leq D_{i} \leq P^{1 / 2}<P$. So if $\mathscr{D}_{J}$ is non-empty, then $|J| \leq m / 4$. Therefore for any triple $\mathscr{D}_{J_{1}}, \mathscr{D}_{J_{2}}, \mathscr{D}_{J_{3}}$ satisfying $\mathscr{D}_{J_{i}} \neq \emptyset(1 \leq i \leq 3)$, there exists $1 \leq j \leq m$ such that $j \notin J_{1} \cup J_{2} \cup J_{3}$. In other words, we have $\left(p, d_{i}\right)=1$ for all $p \in \mathcal{R}_{j}$ and $d_{i} \in \mathscr{D}_{J_{i}}$. 
In view of the square sieve of Heath-Brown [8] and the above preparations, Proposition 4 can be reduced to the estimate

$$
\begin{aligned}
\mathcal{E}_{0} & =\sum_{1 \leq n<N}\left|\sum_{\mathbf{d}} \beta(\mathbf{d})\left(\Omega_{\mathbf{d}}(n)-\mathcal{M}_{\mathbf{d}, Q}(n)\right)\right|^{2}\left(\frac{\log R}{R} \sum_{\substack{p \nmid N \\
R \leq p<2 R}}\left(\frac{N-n}{p}\right)\right)^{2} \\
& \ll P^{4+\varepsilon} R^{-1} Q^{-1}+\left(D_{1} D_{2} D_{3}\right)^{2} P^{2+\varepsilon} R^{3}+P^{\varepsilon} Q^{14 / 5} R^{29 / 5}
\end{aligned}
$$

where $\beta(\mathbf{d})$ is supported on $d_{i} \in \mathscr{D}_{i} \subseteq\left[D_{i}, 2 D_{i}\right), 1 \leq i \leq 3$, satisfying (2.6), (2.7), (2.8), and

$$
\left(d_{1} d_{2} d_{3}, p\right)=1 \text { for all } d_{i} \in \mathscr{D}_{i}, R \leq p<2 R,
$$

and $R$ is restricted by

$$
P^{1+\varepsilon} Q^{-1}<R<P^{2-\varepsilon} Q^{-2} .
$$

Although our choices will be of type $Q^{1-\varepsilon}$ and $R=P^{\varepsilon}$, there is really some space for the parameters $Q$ and $R$ so that $\mathcal{E}_{0} \ll P^{3-\varepsilon}$ and (5.4) hold simultaneously.

\section{Proof of Proposition 4}

We start to estimate $\mathcal{E}_{0}$ which is bounded by

$$
\varepsilon_{0} \ll \frac{\log ^{2} R}{R^{2}}\left|E_{1}\right|+\frac{\log R}{R} \varepsilon_{2},
$$

where

$$
\mathcal{E}_{1}=\sum_{\mathcal{R}}\left(\frac{N-n}{p p^{\prime}}\right) \sum_{1 \leq n<N}\left|\sum_{\mathbf{d}} \beta(\mathbf{d})\left(\Omega_{\mathbf{d}}(n)-\mathcal{M}_{\mathbf{d}, Q}(n)\right)\right|^{2}
$$

and

$$
\mathcal{E}_{2}=\sum_{1 \leq n<N}\left|\sum_{\mathbf{d}} \beta(\mathbf{d})\left(\Omega_{\mathbf{d}}(n)-\mathcal{M}_{\mathbf{d}, Q}(n)\right)\right|^{2} .
$$

Throughout, we use $\sum_{\mathcal{R}}$ to indicate that the summation is taken over prime numbers $p, p^{\prime}$ with $R \leq p \neq p^{\prime}<2 R$ and $\left(p p^{\prime}, N\right)=1$.

We expand the square to obtain

$$
\varepsilon_{2}=\varepsilon_{2}^{(1)}-2 \varepsilon_{2}^{(2)}+\varepsilon_{2}^{(3)}
$$

where

$$
\mathcal{E}_{2}^{(i)}=\sum_{\mathbf{d}} \sum_{\mathbf{t}} \beta(\mathbf{d}) \beta(\mathbf{t}) J_{2}^{(i)}
$$

with

$$
\begin{aligned}
& J_{2}^{(1)}=\int_{0}^{1} f_{\mathbf{d}}(\alpha) f_{\mathbf{t}}(-\alpha) d \alpha, \\
& J_{2}^{(2)}=\int_{0}^{1} f_{\mathbf{d}}(\alpha) W_{\mathbf{t}, Q}(-\alpha) d \alpha, \\
& J_{2}^{(3)}=\int_{0}^{1} W_{\mathbf{d}, Q}(\alpha) W_{\mathbf{t}, Q}(-\alpha) d \alpha .
\end{aligned}
$$


Let

$$
\widetilde{T}_{2}(q, \mathbf{d}, \mathbf{t}, \mathbf{n}, \mathbf{l})=\sum_{a(q)^{*}} \int_{\mathcal{B}(q, a)} S_{\mathbf{d}}(q, a, \mathbf{n}) S_{\mathbf{t}}(q,-a,-\mathbf{l}) I_{\mathbf{d}}\left(\beta N,-\frac{P}{q} \mathbf{n}\right) I_{\mathbf{t}}\left(-\beta N, \frac{P}{q} \mathbf{l}\right) d \beta,
$$

where

$$
\mathscr{B}(q, a)=\left[-\frac{1}{q\left(q+q^{\prime}\right)}, \frac{1}{q\left(q+q^{\prime \prime}\right)}\right]
$$

with $q^{\prime}$ and $q^{\prime \prime}$ satisfying

$$
P<q+q^{\prime}, q+q^{\prime \prime} \leq q+P, \quad a q^{\prime} \equiv 1(\bmod q), \quad a q^{\prime \prime} \equiv-1(\bmod q) .
$$

Set

$$
\mathcal{T}_{2}(q, \mathbf{d}, \mathbf{t})=\mathcal{T}_{2}(q, \mathbf{d}, \mathbf{t}, \mathbf{0}, \mathbf{0})
$$

By Lemma 6, we have

$$
\begin{aligned}
J_{2}^{(3)} & =\sum_{q \leq P} \sum_{a(q)^{*}} \int_{\beta \in B(q, a)} \mathcal{W}_{\mathbf{d}, Q}\left(\frac{a}{q}+\beta\right) \mathcal{W}_{\mathbf{t}, Q}\left(-\frac{a}{q}-\beta\right) d \beta \\
& =\frac{P^{6}}{d_{1} d_{2} d_{3} t_{1} t_{2} t_{3}} \sum_{q \leq Q} \frac{1}{q^{6}} \mathcal{T}_{2}(q, \mathbf{d}, \mathbf{t})+O\left(P^{-A}\right) .
\end{aligned}
$$

So

$$
\varepsilon_{2}^{(3)}=\varepsilon_{2}^{\prime}(Q)+O\left(P^{-A}\right),
$$

where

$$
\varepsilon_{2}^{\prime}(K)=P^{6} \sum_{\mathbf{d}} \sum_{\mathbf{t}} \frac{\beta(\mathbf{d}) \beta(\mathbf{t})}{d_{1} d_{2} d_{3} t_{1} t_{2} t_{3}} \sum_{q \leq K} \frac{1}{q^{6}} \tau_{2}(q, \mathbf{d}, \mathbf{t}) .
$$

According to Lemma 5, one has

$$
J_{2}^{(1)}=\frac{P^{6}}{d_{1} d_{2} d_{3} t_{1} t_{2} t_{3}} \sum_{q \leq P} \frac{1}{q^{6}} \sum_{\mathbf{n} \in \mathcal{N}_{\mathbf{d}, q}} \sum_{\mathbf{l} \in \mathcal{N}_{\mathbf{t}, q}} \mathcal{T}_{2}(q, \mathbf{d}, \mathbf{t}, \mathbf{n}, \mathbf{l})+O\left(P^{-A}\right) .
$$

Combining Lemma 5 and Lemma 6, we derive

$$
J_{2}^{(2)}=\frac{P^{6}}{d_{1} d_{2} d_{3} t_{1} t_{2} t_{3}} \sum_{q \leq Q} \frac{1}{q^{6}} \sum_{\mathbf{n} \in \mathcal{N}_{\mathbf{d}, q}} \mathcal{T}_{2}(q, \mathbf{d}, \mathbf{t}, \mathbf{n}, \mathbf{0})+O\left(P^{-A}\right) .
$$

We exchange the summation over $a$ and the integration by the standard technique to get

$$
\begin{gathered}
\mathcal{T}_{2}(q, \mathbf{d}, \mathbf{t}, \mathbf{n}, \mathbf{l})=\int_{|\beta| \leq \frac{1}{q P}} \sum_{|v| \leq P} \sigma(v ; \beta, q) \sum_{a(q)^{*}} e_{q}(\bar{a} v) S_{\mathbf{d}}(q, a, \mathbf{n}) S_{\mathbf{t}}(q,-a,-\mathbf{l}) \\
\times I_{\mathbf{d}}\left(\beta N,-\frac{P}{q} \mathbf{n}\right) I_{\mathbf{t}}\left(-\beta N, \frac{P}{q} \mathbf{l}\right) d \beta
\end{gathered}
$$

where the function $\sigma$ satisfies $\sigma(v ; \beta, q) \ll 1 /(1+|v|)$. For this technique, one may refer to [9, (98) and (99)], for example. One can also refer to Estermann [5, proof of Lemma 13]. 
Recalling (4.1), we have

$$
\begin{aligned}
\mathcal{T}_{2}(q, \mathbf{d}, \mathbf{t}, \mathbf{n}, \mathbf{l}) \ll & \ll \sum_{|v| \leq P} \frac{1}{1+|v|} \int_{|\beta| \leq \frac{1}{q P}}|\theta(q ; \mathbf{d}, \mathbf{t}, \mathbf{n}, \mathbf{l}, v, 1,1)| \\
& \times\left|I_{\mathbf{d}}\left(\beta N,-\frac{P}{q} \mathbf{n}\right) I_{\mathbf{t}}\left(-\beta N, \frac{P}{q} \mathbf{l}\right)\right| d \beta \\
\leq & \sum_{|v| \leq P} \frac{1}{1+|v|}|\theta(q ; \mathbf{d}, \mathbf{t}, \mathbf{n}, \mathbf{l}, v, 1,1)| \mathcal{d}_{2}(q, \mathbf{d}, \mathbf{t}, \mathbf{n}, \mathbf{l}),
\end{aligned}
$$

where

$$
d_{2}(q, \mathbf{d}, \mathbf{t}, \mathbf{n}, \mathbf{l})=\int_{-\infty}^{\infty}\left|I_{\mathbf{d}}\left(\beta N,-\frac{P}{q} \mathbf{n}\right) I_{\mathbf{t}}\left(-\beta N, \frac{P}{q} \mathbf{l}\right)\right| d \beta .
$$

Let

$$
\begin{aligned}
x_{2}=P^{6} \sum_{|v| \leq P} \frac{1}{1+|v|} \sum_{\mathbf{d}, \mathbf{t}} \frac{|\beta(\mathbf{d}) \beta(\mathbf{t})|}{d_{1} d_{2} d_{3} t_{1} t_{2} t_{3}} \\
\quad \times \sum_{q \leq P} \frac{1}{q^{6}} \sum_{\substack{\mathbf{n} \in \mathcal{N}_{\mathbf{d}, q}, \mathbf{l} \in \mathcal{N}_{\mathbf{t}, q} \\
(\mathbf{n}, \mathbf{l}) \neq \mathbf{0} \in \mathbb{Z}^{6}}}|\theta(q ; \mathbf{d}, \mathbf{t}, \mathbf{n}, \mathbf{l}, v, 1,1)| d_{2}(q, \mathbf{d}, \mathbf{t}, \mathbf{n}, \mathbf{l}) .
\end{aligned}
$$

By (6.3), (6.5), (6.6) and (6.9), we get

$$
\varepsilon_{2}^{(1)}=\varepsilon_{2}^{\prime}(P)+O\left(X_{2}\right)+O\left(P^{-A}\right) .
$$

Similarly from (6.3), (6.5), (6.7) and (6.9) we deduce that

$$
\varepsilon_{2}^{(2)}=\varepsilon_{2}^{\prime}(Q)+O\left(X_{2}\right)+O\left(P^{-A}\right) .
$$

One has, by Lemma 1 ,

$$
\sum_{a(q)^{*}} \int_{\beta \in \mathcal{B}(q, a)} S_{\mathbf{d}}(q, a) S_{\mathbf{t}}(q,-a)|I(\beta N)|^{6} d \beta \ll P^{-2} q^{4}\left(q, \mathbf{d}^{2}\right)^{1 / 2}\left(q, \mathbf{t}^{2}\right)^{1 / 2} .
$$

This yields

$$
\begin{aligned}
\mathcal{E}_{2}^{\prime}(P)-\mathcal{E}_{2}^{\prime}(Q) & \ll P^{4} \sum_{\mathbf{d}} \sum_{\mathbf{t}} \frac{|\beta(\mathbf{d}) \beta(\mathbf{t})|}{d_{1} d_{2} d_{3} t_{1} t_{2} t_{3}} \sum_{Q<q \leq P} \frac{1}{q^{2}}\left(q, \mathbf{d}^{2}\right)^{1 / 2}\left(q, \mathbf{t}^{2}\right)^{1 / 2} \\
& \ll Q^{-1} P^{4+\varepsilon} .
\end{aligned}
$$

We conclude from (6.2), (6.4), (6.10), (6.11) and (6.12) that

$$
\varepsilon_{2} \ll Q^{-1} P^{4+\varepsilon}+X_{2} \text {. }
$$

From Lemma 4 (i),

$$
d_{2}(q, \mathbf{d}, \mathbf{t}, \mathbf{n}, \mathbf{l}) \ll P^{-2+\varepsilon} \min \left\{\frac{q^{2}}{P^{2}}\left(\sum_{j=1}^{3}\left(\frac{\left|n_{j}\right|}{d_{j}}+\frac{\left|l_{i}\right|}{t_{i}}\right)\right)^{-2}, \frac{q}{P}\left(\sum_{j=1}^{3}\left(\frac{\left|n_{j}\right|}{d_{j}}+\frac{\left|l_{i}\right|}{t_{i}}\right)\right)^{-1}\right\} .
$$

Applying Lemma 14, we see that

$$
X_{2} \ll P^{4+\varepsilon}\left(D_{1} D_{2} D_{3}\right)^{-2} y_{2} \ll P^{3+\varepsilon}+\left(D_{1} D_{2} D_{3}\right)^{2} P^{2+\varepsilon} .
$$

Hence our final estimate for $\mathcal{E}_{2}$ is

$$
\varepsilon_{2} \ll P^{4+\varepsilon} Q^{-1}+\left(D_{1} D_{2} D_{3}\right) P^{2+\varepsilon} .
$$


Now we consider $\mathcal{E}_{1}$. Following the argument in [9, Section 3.4.1], we arrive at

$$
\varepsilon_{1}=\varepsilon_{1}^{(1)}-2 \varepsilon_{1}^{(2)}+\varepsilon_{1}^{(3)},
$$

where

$$
\mathcal{E}_{1}^{(i)}=\sum_{\mathcal{R}} \frac{\gamma\left(p p^{\prime}\right)}{p p^{\prime}} \sum_{\mathbf{d}} \sum_{\mathbf{t}} \beta(\mathbf{d}) \beta(\mathbf{t}) \sum_{s\left(p p^{\prime}\right)^{*}}\left(\frac{s}{p p^{\prime}}\right) e_{p p^{\prime}}(-s N) J_{1}^{(i)}
$$

with

$$
\begin{aligned}
J_{1}^{(1)} & =\int_{0}^{1} f_{\mathbf{d}}\left(\alpha+\frac{s}{p p^{\prime}}\right) f_{\mathbf{t}}(-\alpha) d \alpha, \\
J_{1}^{(2)} & =\int_{0}^{1} f_{\mathbf{d}}\left(\alpha+\frac{s}{p p^{\prime}}\right) \mathcal{W}_{\mathbf{t}, Q}(-\alpha) d \alpha, \\
J_{1}^{(3)} & =\sum_{n \in \mathbb{Z}} \mathcal{M}_{\mathbf{d}, Q}(n) \mathcal{M}_{\mathbf{t}, Q}(n) e_{p p^{\prime}}(s n) .
\end{aligned}
$$

We decompose the integral

$$
\begin{aligned}
J_{1}^{(1)} & =\sum_{q \leq P} \sum_{a(q)^{*}} \int_{\beta \in \mathcal{B}(q, a)} f_{\mathbf{d}}\left(\beta+\frac{a}{q}+\frac{s}{p p^{\prime}}\right) f_{\mathbf{t}}\left(-\beta-\frac{a}{q}\right) d \beta \\
& =\sum_{q \leq P} \sum_{\Delta} \sum_{\substack{a(q)^{*} \\
\left(a p p^{\prime}+s q, q p p^{\prime}\right)=\Delta}} \int_{\mathcal{B}(q, a)} f_{\mathbf{d}}\left(\beta+\frac{\left(a p p^{\prime}+s q\right) \Delta^{-1}}{q p p^{\prime} \Delta^{-1}}\right) f_{\mathbf{t}}\left(-\beta-\frac{a}{q}\right) d \beta .
\end{aligned}
$$

Note that $\left(a p p^{\prime}+s q, q p p^{\prime}\right) \mid\left(p p^{\prime}\right)^{2}$, we deduce by Lemma 5

$$
\begin{aligned}
J_{1}^{(1)}= & \sum_{\Delta \mid\left(p p^{\prime}\right)^{2}} \sum_{q \leq P} \frac{P^{6} \Delta^{3}}{q^{6} d_{1} d_{2} d_{3} t_{1} t_{2} t_{3}\left(p p^{\prime}\right)^{3}} \\
& \times \sum_{\mathbf{n} \in \mathcal{N}_{\mathbf{d}, q}\left(p p^{\prime} / \Delta\right)} \sum_{\mathbf{l} \in \mathcal{N}_{\mathbf{t}, q}} \sum_{\substack{a(q)^{*} \\
\left(a p p^{\prime}+s q, q p p^{\prime}\right)=\Delta}} \int_{\mathcal{B}(q, a)} S_{\mathbf{d}}\left(\frac{q p p^{\prime}}{\Delta}, \frac{a p p^{\prime}+s q}{\Delta}, \mathbf{n}\right) \\
& \times S_{\mathbf{t}}(q,-a,-\mathbf{l}) I_{\mathbf{d}}\left(\beta N,-\frac{P \Delta}{q p p^{\prime}} \mathbf{n}\right) I_{\mathbf{t}}\left(-\beta N, \frac{P}{q} \mathbf{l}\right) d \beta+O\left(P^{-A}\right) .
\end{aligned}
$$

Let

$$
\begin{aligned}
\widetilde{T}_{1}\left(q, \mathbf{d}, \mathbf{t}, \mathbf{n}, \mathbf{l}, p, p^{\prime}, \Delta\right)=\sum_{s\left(p p^{\prime}\right)^{*}} & \left(\frac{s}{p p^{\prime}}\right) e_{p p^{\prime}}(-s N) \sum_{a(q)^{*}} \int_{\mathcal{B}(q, a)} S_{\mathbf{d}}\left(\frac{q p p^{\prime}}{\Delta}, \frac{a p p^{\prime}+s q}{\Delta}, \mathbf{n}\right) \\
& \times S_{\mathbf{t}}(q,-a,-\mathbf{l}) I_{\mathbf{d}}\left(\beta N,-\frac{P \Delta}{q p p^{\prime}} \mathbf{n}\right) I_{\mathbf{t}}\left(-\beta N, \frac{P}{q} \mathbf{l}\right) d \beta,
\end{aligned}
$$

and let

$$
\mathcal{T}_{1}\left(q, \mathbf{d}, \mathbf{t}, p, p^{\prime}, \Delta\right)=\mathcal{T}_{1}\left(q, \mathbf{d}, \mathbf{t}, \mathbf{0}, \mathbf{0}, p, p^{\prime}, \Delta\right) .
$$

From above we obtain

$$
\begin{aligned}
\mathcal{E}_{1}^{(1)}=P^{6} & \sum_{\mathcal{R}} \frac{\gamma\left(p p^{\prime}\right)}{\left(p p^{\prime}\right)^{4}} \sum_{\mathbf{d}, \mathbf{t}} \frac{\beta(\mathbf{d}) \beta(\mathbf{t})}{d_{1} d_{2} d_{3} t_{1} t_{2} t_{3}} \\
& \times \sum_{\Delta \mid\left(p p^{\prime}\right)^{2}} \sum_{q \leq P} \frac{\Delta^{3}}{q^{6}} \sum_{\mathbf{n} \in \mathcal{N}_{\mathbf{d}, q}\left(p p^{\prime} / \Delta\right)} \sum_{\mathbf{l} \in \mathcal{N}_{\mathbf{t}, q}} \mathcal{T}_{1}\left(q, \mathbf{d}, \mathbf{t}, \mathbf{n}, \mathbf{l}, p, p^{\prime}, \Delta\right) .
\end{aligned}
$$


By interchanging the summation over $a$ and the integration, we see that $\mathcal{T}_{1}\left(q, \mathbf{d}, \mathbf{t}, \mathbf{n}, \mathbf{l}, p, p^{\prime}, \Delta\right)$ is equal to

$$
\begin{aligned}
\int_{|\beta| \leq \frac{1}{q P}} & \sum_{|v| \leq P} \sigma(v ; q, \beta) \sum_{\begin{array}{c}
a(q)^{*} \\
\left(a p p^{\prime}+s q, q p p^{\prime}\right)=\Delta
\end{array}} e_{q}(\bar{a} v) S_{\mathbf{d}}\left(\frac{q p p^{\prime}}{\Delta}, \frac{a p p^{\prime}+s q}{\Delta}, \mathbf{n}\right) S_{\mathbf{t}}(q,-a,-\mathbf{l}) \\
& \times I_{\mathbf{d}}\left(\beta N,-\frac{P \Delta}{q p p^{\prime}} \mathbf{n}\right) I_{\mathbf{t}}\left(-\beta N, \frac{P}{q} \mathbf{l}\right) d \beta .
\end{aligned}
$$

Recalling the definition of $W\left(\Delta, q ; \mathbf{d}, \mathbf{t}, \mathbf{n}, \mathbf{l}, p, p^{\prime}, v\right)$, we have

$$
\begin{aligned}
& \mathcal{T}_{1}\left(q, \mathbf{d}, \mathbf{t}, \mathbf{n}, \mathbf{l}, p, p^{\prime}, \Delta\right) \\
& \quad \ll \sum_{|v| \leq P} \frac{1}{1+|v|}\left|W\left(\Delta, q ; \mathbf{d}, \mathbf{t}, \mathbf{n}, \mathbf{l}, p, p^{\prime}, v\right)\right| \mathcal{\ell}_{1}\left(q, \mathbf{d}, \mathbf{t}, \mathbf{n}, \mathbf{l}, \frac{p p^{\prime}}{\Delta}\right),
\end{aligned}
$$

where

$$
\iota_{1}(q, \mathbf{d}, \mathbf{t}, \mathbf{n}, \mathbf{l}, H)=\int_{-\infty}^{\infty}\left|I_{\mathbf{d}}\left(\beta N,-\frac{P}{q H} \mathbf{n}\right) I_{\mathbf{t}}\left(-\beta N, \frac{P}{q} \mathbf{l}\right)\right| d \beta .
$$

To discuss the contribution from $(\mathbf{n}, \mathbf{l})=\mathbf{0} \in \mathbb{Z}^{6}$, we define

$$
\begin{aligned}
\mathcal{T}_{1}^{\prime}\left(q, \mathbf{d}, \mathbf{t}, p, p^{\prime}, \Delta\right)= & \sum_{s\left(p p^{\prime}\right)^{*}}\left(\frac{s}{p p^{\prime}}\right) e_{p p^{\prime}}(-s N) \\
& \times \sum_{a(q)^{*}} \int_{|\beta| \leq \frac{1}{2 q P}} S_{\mathbf{d}}\left(\frac{q p p^{\prime}}{\Delta}, \frac{a p p^{\prime}+s q}{\Delta}\right) S_{\mathbf{t}}(q,-a)|I(\beta)|^{6} d \beta \\
= & W\left(\Delta, q ; \mathbf{d}, \mathbf{t}, \mathbf{0}, \mathbf{0}, p, p^{\prime}, 0\right) \int_{|\beta| \leq \frac{1}{2 q P}}|I(\beta)|^{6} d \beta .
\end{aligned}
$$

We deduce from Lemma 4 (ii) that

$$
\begin{aligned}
& \mathcal{T}_{1}^{\prime}\left(q, \mathbf{d}, \mathbf{t}, p, p^{\prime}, \Delta\right)-\mathcal{T}_{1}\left(q, \mathbf{d}, \mathbf{t}, p, p^{\prime}, \Delta\right) \\
& \quad \ll P^{-4} q^{2} \sum_{|v| \leq P} \frac{1}{1+|v|}\left|W\left(\Delta, q ; \mathbf{d}, \mathbf{t}, \mathbf{0}, \mathbf{0}, p, p^{\prime}, v\right)\right|
\end{aligned}
$$

and

$$
\begin{gathered}
\mathcal{T}_{1}^{\prime}\left(q, \mathbf{d}, \mathbf{t}, p, p^{\prime}, \Delta\right)-\sigma_{0} P^{-2} W\left(\Delta, q ; \mathbf{d}, \mathbf{t}, \mathbf{0}, \mathbf{0}, p, p^{\prime}, 0\right) \\
\ll P^{-4} q^{2}\left|W\left(\Delta, q ; \mathbf{d}, \mathbf{t}, \mathbf{0}, \mathbf{0}, p, p^{\prime}, 0\right)\right| .
\end{gathered}
$$

Let

$$
\mathcal{E}_{1}^{\prime}(K)=\sigma_{0} P^{4} \sum_{\mathcal{R}} \frac{\gamma\left(p p^{\prime}\right)}{\left(p p^{\prime}\right)^{4}} \sum_{\mathbf{d}, \mathbf{t}} \frac{\beta(\mathbf{d}) \beta(\mathbf{t})}{d_{1} d_{2} d_{3} t_{1} t_{2} t_{3}} \sum_{\Delta \mid\left(p p^{\prime}\right)^{2}} \sum_{q \leq K} \frac{\Delta^{3}}{q^{6}} W\left(\Delta, q ; \mathbf{d}, \mathbf{t}, \mathbf{0}, \mathbf{0}, p, p^{\prime}, 0\right),
$$

and define

$$
\begin{aligned}
X_{1}=P^{4} R^{-7} & \sum_{\mathcal{R}} \sum_{|v| \leq P} \frac{1}{1+|v|} \sum_{\mathbf{d}, \mathbf{t}} \frac{|\beta(\mathbf{d}) \beta(\mathbf{t})|}{d_{1} d_{2} d_{3} t_{1} t_{2} t_{3}} \sum_{\Delta \mid\left(p p^{\prime}\right)^{2}} \sum_{q \leq P} \frac{\Delta^{3}}{q^{6}} \\
\times & \sum_{\mathbf{n} \in \mathcal{N}_{\mathbf{d}, q}\left(p p^{\prime} / \Delta\right)} \sum_{\mathbf{l} \in \mathcal{N}_{\mathbf{t}, q}}\left|W\left(\Delta, q ; \mathbf{d}, \mathbf{t}, \mathbf{n}, \mathbf{l}, p, p^{\prime}, v\right)\right| e\left(q, \mathbf{d}, \mathbf{t}, \mathbf{n}, \mathbf{l}, \frac{p p^{\prime}}{\Delta}\right) .
\end{aligned}
$$


Here the function $\mathcal{C}\left(q, \mathbf{d}, \mathbf{t}, \mathbf{n}, \mathbf{l}, p p^{\prime} / \Delta\right)$ is defined in (4.6). By Lemma 4 (i), if (n, l) $\neq \mathbf{0} \in \mathbb{Z}^{6}$, then

$$
\iota_{1}\left(q, \mathbf{d}, \mathbf{t}, \mathbf{n}, \mathbf{l}, \frac{p p^{\prime}}{\Delta}\right) \ll P^{-2+\varepsilon} \varphi\left(q, \mathbf{d}, \mathbf{t}, \mathbf{n}, \mathbf{l}, \frac{p p^{\prime}}{\Delta}\right) .
$$

We conclude from (6.16), (6.17), (6.18), (6.19) and (6.20) that

$$
\varepsilon_{1}^{(1)}=\varepsilon_{1}^{\prime}(P)+O\left(\mathcal{X}_{1}\right)+O\left(P^{-A}\right) .
$$

Similarly we can also obtain

$$
\varepsilon_{1}^{(2)}=\varepsilon_{1}^{\prime}(Q)+O\left(\mathcal{X}_{1}\right)+O\left(P^{-A}\right) .
$$

We shall prove

$$
\varepsilon_{1}^{(3)}=\varepsilon_{1}^{\prime}+O\left(P^{\varepsilon} Q^{3} R^{3}\right)+O\left(P^{\varepsilon} Q^{14 / 5} R^{29 / 5}\right),
$$

where

$$
\begin{aligned}
\mathcal{E}_{1}^{\prime}=\sigma_{0} P^{4} & \sum_{\mathcal{R}} \frac{\gamma\left(p p^{\prime}\right)}{\left(p p^{\prime}\right)^{4}} \sum_{\mathbf{d}, \mathbf{t}} \frac{\beta(\mathbf{d}) \beta(\mathbf{t})}{d_{1} d_{2} d_{3} t_{1} t_{2} t_{3}} \\
& \times \sum_{\Delta \mid\left(p p^{\prime}\right)^{2}} \Delta^{3} \sum_{q \leq \min \left\{Q, Q \Delta\left(p p^{\prime}\right)^{-1}\right\}} \frac{1}{q^{6}} W\left(\Delta, q ; \mathbf{d}, \mathbf{t}, \mathbf{0}, \mathbf{0}, p, p^{\prime}, 0\right) .
\end{aligned}
$$

Note that $\min \left\{Q, Q \Delta\left(p p^{\prime}\right)^{-1}\right\} \leq Q<P$, we introduce

$$
\begin{aligned}
x_{3}=P^{4} R^{-7} & \sum_{\mathcal{R}} \sum_{\mathbf{d}, \mathbf{t}} \frac{|\beta(\mathbf{d}) \beta(\mathbf{t})|}{d_{1} d_{2} d_{3} t_{1} t_{2} t_{3}} \\
& \times \sum_{\Delta \mid\left(p p^{\prime}\right)^{2}} \sum_{\min \left\{Q, Q \Delta\left(p p^{\prime}\right)^{-1}\right\} \leq q \leq P} \frac{\Delta^{3}}{q^{6}}\left|W\left(\Delta, q ; \mathbf{d}, \mathbf{t}, \mathbf{0}, \mathbf{0}, p, p^{\prime}, 0\right)\right| .
\end{aligned}
$$

Thus by (6.14), (6.21), (6.22) and (6.23) we arrive at

$$
\varepsilon_{1} \ll O\left(\mathcal{X}_{1}\right)+O\left(\mathcal{X}_{3}\right)+O\left(P^{\varepsilon} Q^{3} R^{3}\right)+O\left(P^{\varepsilon} Q^{14 / 5} R^{29 / 5}\right) .
$$

By Lemma 14, we have

$$
x_{1} \ll P^{4+\varepsilon} R^{-7}\left(D_{1} D_{2} D_{3}\right)^{-2} \sum_{\mathcal{R}} y_{1} \ll P^{3+\varepsilon} R+\left(D_{1} D_{2} D_{3}\right)^{2} P^{2+\varepsilon} R^{5} .
$$

From Lemma 11,

$$
\begin{aligned}
& W\left(\Delta, q ; \mathbf{d}, \mathbf{t}, \mathbf{0}, \mathbf{0}, p, p^{\prime}, v\right) \\
& \quad \ll 2^{4 u} r^{4}\left(r, \mathbf{d}^{2}\right)^{1 / 2}\left(r, \mathbf{t}^{2}\right)^{1 / 2} \Delta^{-2} R^{4}\left(\pi \pi^{\prime}\right)^{5}\left(1+\Delta R^{-2}\right)^{-1} .
\end{aligned}
$$

Substituting (6.24) into the definition of $\mathcal{X}_{3}$, we can deduce that

$$
\begin{aligned}
x_{3} & \ll P^{4} R^{-3} \sum_{\mathcal{R}} \sum_{\mathbf{d}, \mathbf{t}} \frac{|\beta(\mathbf{d}) \beta(\mathbf{t})|}{d_{1} d_{2} d_{3} t_{1} t_{2} t_{3}} \\
& \times \sum_{\Delta \mid\left(p p^{\prime}\right)^{2}} \sum_{\min \left\{Q, Q \Delta\left(p p^{\prime}\right)^{-1}\right\} \leq 2^{u} \pi \pi^{\prime} r} \frac{\Delta\left(r, \mathbf{d}^{2}\right)^{1 / 2}\left(r, \mathbf{t}^{2}\right)^{1 / 2}}{2^{2 u} \pi \pi^{\prime} r^{2}\left(1+\Delta R^{-2}\right)} \\
& \ll P^{4+\varepsilon} R^{-3} \sum_{\mathcal{R}} \sum_{\Delta \mid\left(p p^{\prime}\right)^{2}} \Delta \min \left\{Q, Q \Delta\left(p p^{\prime}\right)^{-1}\right\}^{-1}\left(1+\Delta R^{-2}\right)^{-1} \\
& \ll P^{4+\varepsilon} R Q^{-1} .
\end{aligned}
$$


Thus for $\mathscr{E}_{1}$ we finally obtain

$$
\mathcal{E}_{1} \ll P^{4+\varepsilon} R Q^{-1}+\left(D_{1} D_{2} D_{3}\right)^{2} P^{2+\varepsilon} R^{5}+P^{\varepsilon} Q^{14 / 5} R^{29 / 5} .
$$

Combining (6.13), (6.25) and the lines around (5.3) and (6.1), we established Proposition 4.

We are left to establish (6.23). Recalling the definition of $J_{1}^{(3)}$, one has

$$
\begin{gathered}
J_{1}^{(3)}=\frac{P^{2}}{d_{1} d_{2} d_{3} t_{1} t_{2} t_{3}} \sum_{q_{1}, q_{2} \leq Q}\left(q_{1} q_{2}\right)^{-3} \sum_{\substack{a_{1}\left(q_{1}\right)^{*} \\
a_{2}\left(q_{2}\right)^{*}}} S_{\mathbf{d}}\left(q_{1},-a_{1}\right) S_{\mathbf{t}}\left(q_{2},-a_{2}\right) \\
\times \sum_{n \in Z} H^{2}\left(\frac{n}{N}\right) e\left(n\left(\frac{a_{1}}{q_{1}}+\frac{a_{2}}{q_{2}}+\frac{s}{p p^{\prime}}\right)\right) .
\end{gathered}
$$

By Lemma 7, we get

$$
\varepsilon_{1}^{(3)}=\varepsilon_{1}^{\prime \prime}+E+O\left(P^{-A}\right)
$$

where

$$
\begin{aligned}
\mathcal{E}_{1}^{\prime \prime}=\sigma_{0} P^{4} & \sum_{\mathcal{R}} \frac{\gamma\left(p p^{\prime}\right)}{p p^{\prime}} \sum_{\mathbf{d}} \sum_{\mathbf{t}} \frac{\beta(\mathbf{d}) \beta(\mathbf{t})}{d_{1} d_{2} d_{3} t_{1} t_{2} t_{3}} \sum_{s\left(p p^{\prime}\right)^{*}}\left(\frac{s}{p p^{\prime}}\right) e_{p p^{\prime}}(-s N) \\
& \times \sum_{q_{1}, q_{2} \leq Q}\left(q_{1} q_{2}\right)^{-3} \sum_{\substack{a_{1}\left(q_{1}\right)^{*}, a_{2}\left(q_{2}\right)^{*} \\
\frac{a_{1}}{q_{1}}+\frac{a_{2}}{q_{2}}+\frac{s}{p p^{\prime}} \in \mathbb{Z}}} S_{\mathbf{d}}\left(q_{1},-a_{1}\right) S_{\mathbf{t}}\left(q_{2},-a_{2}\right)
\end{aligned}
$$

and

$$
\begin{aligned}
& E=\sum_{\mathcal{R}} \frac{\gamma\left(p p^{\prime}\right)}{p p^{\prime}} \sum_{\mathbf{d}, \mathbf{t}} \frac{P^{2} \beta(\mathbf{d}) \beta(\mathbf{t})}{d_{1} d_{2} d_{3} t_{1} t_{2} t_{3}} \sum_{n \in \mathbb{Z}} H^{2}\left(\frac{n}{N}\right) \sum_{s\left(p p^{\prime}\right)^{*}}\left(\frac{s}{p p^{\prime}}\right) e_{p p^{\prime}}(-s N) \sum_{q_{1}, q_{2} \leq Q} \frac{1}{q_{1}^{3} q_{2}^{3}} \\
& \times \sum_{\substack{a_{1}\left(q_{1}\right)^{*}, a_{2}\left(q_{2}\right)^{*} \\
0<\left\|\frac{a_{1}}{q_{1}}+\frac{a_{2}}{q_{2}}+\frac{s}{p p^{\prime}}\right\| \leq P^{\varepsilon-2}}} S_{\mathbf{d}}\left(q_{1},-a_{1}\right) S_{\mathbf{t}}\left(q_{2},-a_{2}\right) e\left(n\left(\frac{a_{1}}{q_{1}}+\frac{a_{2}}{q_{2}}+\frac{s}{p p^{\prime}}\right)\right) .
\end{aligned}
$$

Notice that

$$
\frac{a_{1}}{q_{1}}+\frac{a_{2} p p^{\prime}+s q_{2}}{q_{2} p p^{\prime}}=\frac{a_{1}}{q_{1}}+\frac{a_{2}}{q_{2}}+\frac{s}{p p^{\prime}} \in \mathbb{Z}
$$

is equivalent to

$$
q_{1}=q_{2} p p^{\prime} \Delta^{-1} \quad \text { and } \quad-a_{1} \equiv\left(a_{2} p p^{\prime}+s q_{2}\right) \Delta^{-1}\left(\bmod q_{1}\right),
$$

where $\Delta=\left(a_{2} p p^{\prime}+s q_{2}, q_{2} p p^{\prime}\right)$. Hence

$$
\varepsilon_{1}^{\prime \prime}=\varepsilon_{1}^{\prime}
$$

Now we handle $E$. By Lemma 1 (vi),

$$
E \ll P^{4} R \sup _{n, p, p^{\prime}} \sum_{\mathbf{d}} \sum_{\mathbf{t}} \frac{|\beta(\mathbf{d}) \beta(\mathbf{t})|}{d_{1} d_{2} d_{3} t_{1} t_{2} t_{3}}|\mathcal{F}|,
$$


where

$$
\begin{aligned}
\mathscr{F}:=\sum_{s\left(p p^{\prime}\right)^{*}}\left(\frac{s}{p p^{\prime}}\right) e_{p p^{\prime}}(-s N) \sum_{q_{1}, q_{2} \leq Q}\left(q_{1} q_{2}\right)^{-3} \\
\times \sum_{\substack{a_{1}\left(q_{1}\right)^{*}, a_{2}\left(q_{2}\right)^{*} \\
0<\left\|\frac{a_{1}}{q_{1}}+\frac{a_{2}}{q_{2}}+\frac{s}{p p^{\prime}}\right\| \leq P^{\varepsilon-2}}} S_{\mathbf{d}}\left(q_{1},-a_{1}\right) S_{\mathbf{t}}\left(q_{2},-a_{2}\right) e\left(n\left(\frac{a_{1}}{q_{1}}+\frac{a_{2}}{q_{2}}+\frac{s}{p p^{\prime}}\right)\right) .
\end{aligned}
$$

Note that

$$
\frac{a_{1}}{q_{1}}+\frac{a_{2}}{q_{2}}+\frac{s}{p p^{\prime}}=\frac{p p^{\prime}\left(a_{1} q_{2}+a_{2} q_{1}\right)+s q_{1} q_{2}}{q_{1} q_{2} p p^{\prime}} .
$$

We divide the summations according to $p p^{\prime}\left(a_{1} q_{2}+a_{2} q_{1}\right)+s q_{1} q_{2}\left(\bmod q_{1} q_{2} p p^{\prime}\right)$,

$$
\begin{aligned}
& \mathcal{F}=\sum_{0<|l| \leq R^{2} Q^{2}} \sum_{P^{\varepsilon-2}}\left(\frac{s}{s\left(p p^{\prime}\right)^{*}}\right) e_{p p^{\prime}}(-s N) \sum_{\substack{q_{1}, q_{2} \leq Q \\
|l|\left(p p^{\prime}\right)^{-1} P^{2-\varepsilon}<q_{1} q_{2}}}\left(q_{1} q_{2}\right)^{-3} \\
& \times \sum_{a_{1}\left(q_{1}\right)^{*}, a_{2}\left(q_{2}\right)^{*}} S_{\mathbf{d}}\left(q_{1},-a_{1}\right) S_{\mathbf{t}}\left(q_{2},-a_{2}\right) \\
& p p^{\prime}\left(a_{1} q_{2}+a_{2} q_{1}\right)+s q_{1} q_{2} \equiv l\left(\bmod q_{1} q_{2} p p^{\prime}\right) \\
& \times e\left(n\left(\frac{a_{1}}{q_{1}}+\frac{a_{2}}{q_{2}}+\frac{s}{p p^{\prime}}\right)\right) .
\end{aligned}
$$

Here the restriction $0<|l| \leq R^{2} Q^{2} P^{\varepsilon-2}$ and $|l|\left(p p^{\prime}\right)^{-1} P^{2-\varepsilon}<q_{1} q_{2}$ come from

$$
0<\left\|\frac{a_{1}}{q_{1}}+\frac{a_{2}}{q_{2}}+\frac{s}{p p^{\prime}}\right\| \leq P^{\varepsilon-2} .
$$

The congruence $p p^{\prime}\left(a_{1} q_{2}+a_{2} q_{1}\right)+s q_{1} q_{2} \equiv l\left(\bmod q_{1} q_{2} p p^{\prime}\right)$ implies

$$
e\left(n\left(\frac{a_{1}}{q_{1}}+\frac{a_{2}}{q_{2}}+\frac{s}{p p^{\prime}}\right)\right)=e\left(\frac{\ln }{q_{1} q_{2} p p^{\prime}}\right) .
$$

Thus

$$
\begin{aligned}
\mathcal{F}= & \sum_{0<|l| \leq R^{2} Q^{2}} \sum_{P^{\varepsilon-2}}\left(\frac{s}{s\left(p p^{\prime}\right)^{*}}\right) e_{p p^{\prime}}(-s N) \sum_{\substack{q_{1}, q_{2} \leq Q \\
|l|\left(p p^{\prime}\right)^{-1} P^{2-\varepsilon}<q_{1} q_{2}}}\left(q_{1} q_{2}\right)^{-3} \\
& \times e\left(\frac{l n}{q_{1} q_{2} p p^{\prime}}\right) \sum_{\substack{a_{1}\left(q_{1}\right)^{*}, a_{2}\left(q_{2}\right)^{*} \\
p p^{\prime}\left(a_{1} q_{2}+a_{2} q_{1}\right)+s q_{1} q_{2} \equiv l\left(\bmod q_{1} q_{2} p p^{\prime}\right)}} S_{\mathbf{d}\left(q_{1},-a_{1}\right) S_{\mathbf{t}}\left(q_{2},-a_{2}\right) .}
\end{aligned}
$$

Recall the assumption (5.4), we easily know $\left(l, p p^{\prime}\right)=1$. This implies $\left(q_{1} q_{2}, p p^{\prime}\right)=1$. So

$$
p p^{\prime}\left(a_{1} q_{2}+a_{2} q_{1}\right)+s q_{1} q_{2} \equiv l\left(\bmod q_{1} q_{2} p p^{\prime}\right)
$$

is equivalent to

$$
p p^{\prime}\left(a_{1} q_{2}+a_{2} q_{1}\right) \equiv l\left(\bmod q_{1} q_{2}\right) \quad \text { and } \quad s q_{1} q_{2} \equiv l\left(\bmod p p^{\prime}\right)
$$


Thus

$$
\begin{gathered}
\mathscr{F}=\sum_{0<|l| \leq R^{2} Q^{2} P^{\varepsilon-2}} \sum_{\substack{q_{1}, q_{2} \leq Q \\
|l|\left(p p^{\prime}\right)^{-1} P^{2-\varepsilon}<q_{1} q_{2}}}\left(q_{1} q_{2}\right)^{-3}\left(\frac{q_{1} q_{2} l}{p p^{\prime}}\right) e_{p p^{\prime}}\left(-\overline{q_{1} q_{2}} l N\right) \\
\times e\left(\frac{l n}{q_{1} q_{2} p p^{\prime}}\right) \sum_{\substack{a_{1}\left(q_{1}\right)^{*}, a_{2}\left(q_{2}\right)^{*} \\
p p^{\prime}\left(a_{1} q_{2}+a_{2} q_{1}\right) \equiv l\left(\bmod q_{1} q_{2}\right)}} S_{\mathbf{d}\left(q_{1},-a_{1}\right) S_{\mathbf{t}}\left(q_{2},-a_{2}\right) .}
\end{gathered}
$$

Now (6.23) follows from Lemma 15, (6.26), (6.27) and (6.28). The proof of Proposition 4 is complete.

\section{Proof of Proposition 3}

Let us define

$$
\widetilde{\mathscr{H}}\left(D_{1}, D_{2}, D_{3}\right)=\sum_{\substack{d_{i} \leq D_{i}(1 \leq i \leq 3) \\\left(d_{i}, d_{j}\right) \leq P^{\varepsilon}(1 \leq i<j \leq 3)}} \beta(\mathbf{d})\left(\mathscr{L}_{\mathbf{d}}(N)-\frac{\mathcal{N}_{0}(N) \Sigma_{0}(\mathbf{d}, N)}{d_{1} d_{2} d_{3}}\right),
$$

where $\beta(\mathbf{d})=\beta\left(d_{1}, d_{2}, d_{3}\right)$ is a real function satisfying (2.6) and (2.7). We have the following result.

Proposition 5. Suppose that $D_{1} D_{2} D_{3}<P^{1 / 2-\varepsilon}$. We have

$$
\widetilde{\mathscr{H}}\left(D_{1}, D_{2}, D_{3}\right) \ll P^{2}(\log P)^{-A} \text {. }
$$

We first explain that Proposition 3 can be deduced from Proposition 5. In order to prove

$$
\mathscr{H}(D) \ll P^{2}(\log P)^{-A} \text { for } D \leq P^{1 / 2-5 \varepsilon},
$$

we divide the underlying summation into two parts. Note that

$$
\begin{aligned}
& \mathscr{H}(D)=\sum_{\substack{d_{1}, d_{2}, d_{3} \\
\left[d_{1}, d_{2}, d_{3}\right] \leq D}} \beta(\mathbf{d})\left(\mathscr{L}_{\mathbf{d}}(N)-\frac{\mathcal{N}_{0}(N) \Sigma_{0}(\mathbf{d}, N)}{d_{1} d_{2} d_{3}}\right) \\
& =\left(\sum_{\substack{d_{1}, d_{2}, d_{3} \\
\max _{i<j}\left(d_{i}, d_{j}\right) \leq P^{\varepsilon} \\
\left[d_{1}, d_{2}, d_{3}\right] \leq D}}+\sum_{\substack{d_{1}, d_{2}, d_{3} \\
\max _{i<j}\left(d_{i}, d_{j}\right)>P^{\varepsilon} \\
\left[d_{1}, d_{2}, d_{3}\right] \leq D}}\right) \beta(\mathbf{d})\left(\mathscr{L}_{\mathbf{d}}(N)-\frac{\mathcal{N}_{0}(N) \Sigma_{0}(\mathbf{d}, N)}{d_{1} d_{2} d_{3}}\right) \\
& =: \mathscr{H}_{1}(D)+\mathscr{H}_{2}(D) \text {. }
\end{aligned}
$$

Since $\max _{i<j}\left(d_{i}, d_{j}\right) \leq P^{\varepsilon}$ and $\left[d_{1}, d_{2}, d_{3}\right] \leq D$ together imply $d_{1} d_{2} d_{3} \leq P^{1 / 2-2 \varepsilon}$, by the dyadic argument and Proposition 5, we can conclude

$$
\mathscr{H}_{1}(D) \ll P^{2}(\log P)^{-A} .
$$

Now we consider $\mathscr{H}_{2}(D)$ which is bounded by $\mathscr{H}^{\prime} P^{\varepsilon / 9}$, where

$$
\mathscr{H}^{\prime}=\sum_{\substack{d_{j} \leq D \\ \max _{i<j}\left(d_{i}, d_{j}\right) \geq P^{\varepsilon}}}\left|\mathscr{L}_{\mathbf{d}}(N)-\frac{\mathcal{N}_{0}(N) \Sigma_{0}(\mathbf{d}, N)}{d_{1} d_{2} d_{3}}\right| .
$$


It suffices to verify $\mathscr{H}^{\prime} \ll P^{2-\varepsilon / 5}$. By symmetry, the assertion is a consequence of

$$
\mathscr{H}_{1}^{\prime}:=\sum_{\substack{d_{j} \leq D(1 \leq j \leq 3) \\\left(d_{1}, d_{2}\right) \geq P^{\varepsilon}}}\left|\frac{\mathcal{N}_{0}(N) \Sigma_{0}(\mathbf{d}, N)}{d_{1} d_{2} d_{3}}\right| \ll P^{2-\varepsilon / 5}
$$

and

$$
\mathscr{H}_{2}^{\prime}:=\sum_{\substack{d_{j} \leq D(1 \leq j \leq 3) \\\left(d_{1}, d_{2}\right) \geq P^{\varepsilon}}} \mathscr{L}_{\mathbf{d}}(N) \ll P^{2-\varepsilon / 5} .
$$

It has been pointed out in [9] that

$$
\Sigma_{0}(\mathbf{d}, N) \ll \tau^{2}\left(d_{1}^{2}\right) \tau^{2}\left(d_{2}^{2}\right) \tau^{2}\left(d_{3}^{2}\right) \log \log P \quad \text { and } \quad \mathcal{N}_{0}(N) \asymp \frac{P^{2}}{\log P} .
$$

Hence

$$
\mathscr{H}_{1}^{\prime} \ll P^{2+\frac{\varepsilon}{10}} \sum_{\substack{d_{j} \leq D \\
\left(d_{1}, d_{2}\right) \geq P^{\varepsilon}}} \frac{1}{d_{1} d_{2} d_{3}} \ll P^{2+\frac{\varepsilon}{5}} \sum_{\begin{array}{r}
d_{1} \leq D, d_{2} \leq D \\
\left(d_{1}, d_{2}\right) \geq P^{\varepsilon}
\end{array}} \frac{1}{d_{1} d_{2}} .
$$

Note that $\omega(\mathbf{x}) \ll 1$, we have

$$
\mathcal{H}_{2}^{\prime} \ll \sum_{\substack{d_{1} \leq P, d_{2} \leq P \\\left(d_{1}, d_{2}\right) \geq P^{\varepsilon}}} \sum_{\substack{x_{1}, x_{2} \\ d_{1}\left|x_{1}, d_{2}\right| x_{2}}} \sum_{\substack{q, x_{3}, d_{3} \\ q^{2}+x_{1}^{2}+x_{2}^{2}+x_{3}^{2}=N \\ d_{3} \mid x_{3}}} 1 .
$$

For fixed $x_{1}, x_{2}$, the inner sum is bounded by $P^{\varepsilon / 10}$. Hence

$$
\mathcal{H}_{2}^{\prime} \ll P^{\varepsilon / 10} \sum_{\substack{d_{1} \leq P, d_{2} \leq P \\
\left(d_{1}, d_{2}\right) \geq P^{\varepsilon}}} \sum_{\substack{x_{1}, x_{2} \\
d_{1}\left|x_{1}, d_{2}\right| x_{2}}} 1 \ll P^{\varepsilon / 10} \sum_{\begin{array}{c}
d_{1} \leq P, d_{2} \leq P \\
\left(d_{1}, d_{2}\right) \geq P^{\varepsilon}
\end{array}} \frac{P^{2}}{d_{1} d_{2}} .
$$

As an exercise, we have

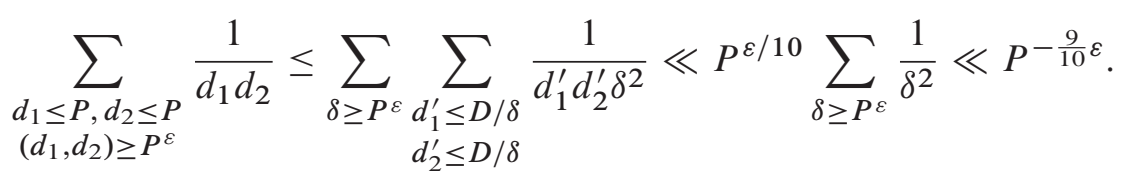

Now the estimates (7.1) and (7.2) follow from (7.3) and (7.4), respectively.

The remaining of this section is to show Proposition 5 by invoking Proposition 4 . The proof follows the argument of Heath-Brown and Tolev closely. Let

$$
\mathscr{H}_{1}=\sum_{\begin{array}{c}
d_{i} \leq D_{i}(1 \leq i \leq 3) \\
\left(d_{i}, d_{j}\right) \leq P^{\varepsilon}(1 \leq i<j \leq 3)
\end{array}} \beta(\mathbf{d}) \mathscr{L}_{\mathbf{d}}(N) .
$$

By applying Proposition 4, we see that

$$
\mathscr{H}_{1}=\sum_{k \in \mathcal{A}_{j}} \omega(k) \sum_{\substack{d_{i} \leq D_{i}(1 \leq i \leq 3) \\\left(d_{i}, d_{j}\right) \leq P^{\varepsilon}(1 \leq i<j \leq 3)}} \beta(\mathbf{d}) \Omega_{\mathbf{d}}\left(N-k^{2}\right)=\mathscr{H}_{2}+O\left(P^{2-\varepsilon}\right),
$$


where

$$
\mathscr{H}_{2}=\sum_{k \in \mathcal{A}_{j}} \omega(k) \sum_{\substack{d_{i} \leq D_{i}(1 \leq i \leq 3) \\\left(d_{i}, d_{j}\right) \leq P^{\varepsilon}(1 \leq i<j \leq 3)}} \beta(\mathbf{d}) \mathcal{M}_{\mathbf{d}}, Q\left(N-k^{2}\right) .
$$

Recalling the definition of $\mathcal{M}_{\mathbf{d}, Q}(n)$, we know

$$
\begin{aligned}
\mathscr{H}_{2}=P & \sum_{d_{i} \leq D_{i}} \frac{\widetilde{\beta}(\mathbf{d})}{d_{1} d_{2} d_{3}} \sum_{q \leq i \leq 2)} q^{-3} \sum_{a(q)^{*}} S_{\mathbf{d}}(q, a) \\
& \times \sum_{k \in \mathcal{A}_{j}} \omega(k) H\left(1-\frac{k^{2}}{N}\right) e\left(\frac{a}{q}\left(k^{2}-N\right)\right),
\end{aligned}
$$

where $\widetilde{\beta}(\mathbf{d})$ is $\beta(\mathbf{d})$ if $\left(d_{i}, d_{j}\right) \leq P^{\varepsilon}(1 \leq i<j \leq 3)$, and zero otherwise. Now partial summation gives

$$
\mathscr{H}_{2}=-P \int \mathscr{B}(x)\left(\frac{d}{d x} \omega(x) H\left(1-\frac{x^{2}}{N}\right)\right) d x,
$$

where

$$
\mathscr{B}(x)=\sum_{d_{i} \leq D_{i}(1 \leq i \leq 3)} \frac{\widetilde{\beta}(\mathbf{d})}{d_{1} d_{2} d_{3}} \sum_{q \leq Q} q^{-3} \sum_{a(q)^{*}} S_{\mathbf{d}}(q, a) \mathcal{Z}(x)
$$

and

$$
\mathcal{Z}(x)=\sum_{\substack{k \leq x \\ k \in \mathcal{A}_{j}}} e\left(\frac{a}{q}\left(k^{2}-N\right)\right)
$$

Let us write

$$
\mathcal{Z}_{0}(x)=\sum_{\substack{k \leq x \\ k \in \mathcal{A}_{j} \\(k, q)=1}} e\left(\frac{a}{q}\left(k^{2}-N\right)\right)
$$

Then we have

$$
\mathcal{Z}(x)=Z_{0}(x)+\sum_{\substack{k \leq x \\ k \in \mathcal{A}_{j} \\(k, q)>1}} e\left(\frac{a}{q}\left(k^{2}-N\right)\right)
$$

and thereby

$$
\mathscr{B}(x)=\mathscr{B}_{0}(x)+\mathbf{E}(x),
$$

where

$$
\mathscr{B}_{0}(x)=\sum_{d_{i} \leq D_{i}(1 \leq i \leq 3)} \frac{\widetilde{\beta}(\mathbf{d})}{d_{1} d_{2} d_{3}} \sum_{q \leq Q} q^{-3} \sum_{a(q)^{*}} S_{\mathbf{d}}(q, a) \mathcal{Z}_{0}(x)
$$

and

$$
\mathbf{E}(x)=\sum_{d_{i} \leq D_{i}(1 \leq i \leq 3)} \frac{\widetilde{\beta}(\mathbf{d})}{d_{1} d_{2} d_{3}} \sum_{q \leq Q} q^{-3} \sum_{a(q)^{*}} S_{\mathbf{d}}(q, a) \sum_{\substack{k \leq x \\ k \in \mathcal{A}_{j} \\(k, q)>1}} e\left(\frac{a}{q}\left(k^{2}-N\right)\right) .
$$


If $(k, q)>1$ and $k \in \mathcal{A}_{j}$, then $(k, q) \geq P^{1 / 16}$. So we have

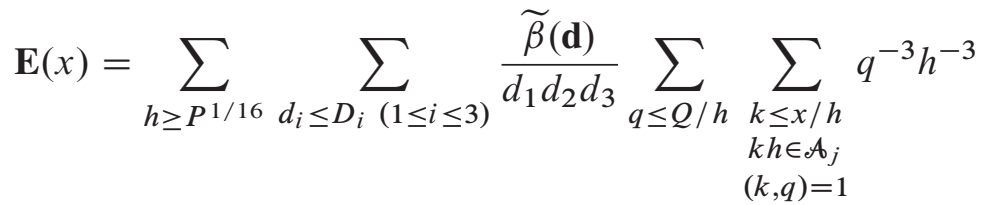

$$
\begin{aligned}
& \times \sum_{a(h q)^{*}} S_{\mathbf{d}}(h q, a) e\left(\frac{a}{h q}\left(k^{2} h^{2}-N\right)\right) .
\end{aligned}
$$

For the inner sum above, we have the bound

$$
\sum_{a(q)^{*}} S_{\mathbf{d}}(q, a) e\left(-\frac{a}{q} n\right) \ll(n, q)\left(q, d_{1}^{2}\right)^{1 / 2}\left(q, d_{2}^{2}\right)^{1 / 2}\left(q, d_{3}^{2}\right)^{1 / 2} q^{2} .
$$

Then we get

$$
\mathbf{E}(x) \ll P^{\varepsilon} \sum_{h \geq P^{1 / 16}} \sum_{q \leq Q / h} q^{-1} h^{-1} \sum_{k \leq x / h}\left(N-k^{2} h^{2}, q h\right) .
$$

We finally find that

$$
\mathbf{E}(x) \ll P^{1-1 / 16+\varepsilon} .
$$

For $(m, q)=1$, we introduce the notation

$$
\Delta_{j}(x ; q, m)=\sum_{\substack{k \leq x \\ k \in \mathcal{A}_{j} \\ k \equiv m(\bmod q)}} 1-\frac{1}{\phi(q)} \Lambda_{j}(x ; q),
$$

where

$$
\Lambda_{j}(x ; q)=\sum_{\substack{k \leq x \\ k \in \mathcal{A}_{j} \\(k, q)=1}} 1
$$

Then we have

$$
\mathfrak{Z}_{0}(x)=\frac{1}{\phi(q)} e\left(\frac{-a N}{q}\right) T(q, a) \Lambda_{j}(x ; q)+\sum_{m(q)^{*}} e\left(\frac{a\left(m^{2}-N\right)}{q}\right) \Delta_{j}(x ; q, m) .
$$

Therefore

$$
\mathscr{B}_{0}(x)=\widetilde{\mathscr{B}}_{0}(x)+\mathscr{C}(x),
$$

where

$$
\widetilde{B}_{0}(x)=\sum_{d_{i} \leq D_{i}} \frac{\widetilde{\beta}(\mathbf{d})}{d_{1} d_{2} d_{3}} \sum_{q \leq 2)} h_{\mathbf{d}}(q) \Lambda_{j}(x ; q)
$$

and

$$
\digamma(x)=\sum_{d_{i} \leq D_{i}(1 \leq i \leq 3)} \frac{\widetilde{\beta}(\mathbf{d})}{d_{1} d_{2} d_{3}} \sum_{q \leq Q} q^{-3} \sum_{a(q)^{*}} S_{\mathbf{d}}(q, a) \sum_{m(q)^{*}} e\left(\frac{a\left(m^{2}-N\right)}{q}\right) \Delta_{j}(x ; q, m) .
$$

Define

$$
L=\sum_{q \leq Q} \sum_{m(q)^{*}} \Delta_{j}(x ; q, m)^{2}
$$


and

$$
M=\sum_{q \leq Q} \sum_{m(q)^{*}} \Gamma(q, m)^{2}
$$

where

$$
\Gamma(q, m)=q^{-3} \sum_{d_{i} \leq D_{i}(1 \leq i \leq 3)} \frac{\widetilde{\beta}(\mathbf{d})}{d_{1} d_{2} d_{3}} \sum_{a(q)^{*}} S_{\mathbf{d}}(q, a) e\left(\frac{a}{q}\left(m^{2}-N\right)\right) .
$$

It has been proved in [9] that $M \ll(\log P)^{C}$ for some absolute constant $C>0$ (see [9, (274)]). The Generalized Barban-Davenport-Halberstam Theorem states that

$$
L \ll P^{2}(\log P)^{-A} .
$$

Observing that

$$
\varphi(x)=\sum_{q \leq Q} \sum_{m(q)^{*}} \Delta_{j}(x ; q, m) \Gamma(q, m),
$$

one can conclude by Cauchy's inequality

$$
\varphi(x) \ll P(\log P)^{-A} .
$$

Let

$$
A(x)=\sum_{\substack{k \leq x \\ k \in \mathscr{A}_{j}}} 1
$$

Note that $\Lambda_{j}(x ; q)=A(x)+O\left(P^{1-1 / 16+\varepsilon}\right)$ and

$$
\sum_{q \leq Q}\left|h_{\mathbf{d}}(q)\right| \ll \tau^{2}\left(d_{1}\right) \tau^{2}\left(d_{2}\right) \tau^{2}\left(d_{3}\right) \log \log P .
$$

Then we have

$$
\widetilde{\mathcal{B}}_{0}(x)=\mathscr{B}_{0} A(x)+O\left(P^{1-1 / 16+\varepsilon}\right),
$$

where

$$
\mathscr{B}_{0}=\sum_{d_{i} \leq D_{i}(1 \leq i \leq 3)} \frac{\widetilde{\beta}(\mathbf{d})}{d_{1} d_{2} d_{3}} \sum_{q \leq Q} h_{\mathbf{d}}(q)
$$

It is not hard to see that

$$
\mathcal{B}_{0}=\mathscr{B}_{1}+O\left(P^{-1 / 2+\varepsilon}\right)
$$

where

$$
\mathscr{B}_{1}=\sum_{d_{i} \leq D_{i}} \frac{\widetilde{\beta}(\mathbf{d})}{d_{1} d_{2} d_{3}} \sum_{q=1}^{\infty} h_{\mathbf{d}}(q) \text {. }
$$

Therefore

$$
\mathscr{B}(x)=\mathscr{B}_{1} A(x)+O\left(P(\log P)^{-A}\right) .
$$

By the Prime Number Theorem, we have

$$
A(x)=\int_{2}^{x} \frac{C_{j}(t)}{\log t} d t+O\left(P(\log P)^{-A}\right) .
$$

Now combining (7.5) and (7.6), we arrive at

$$
\mathscr{H}_{2}=P \mathscr{B}_{1} \int \omega(x) H\left(1-\frac{x^{2}}{N}\right) \frac{C_{j}(x)}{\log x} d x+O\left(P^{2}(\log P)^{-A}\right) .
$$

The proof of Proposition 5 is completed and therefore Proposition 3 is also established. 


\section{The review of the three-dimensional sieve}

In this section, we recall the Diamond-Halberstam-Richert sieves. One may refer to [7] for the details. We shall focus on the case that the sieve dimension $\kappa=3$. Let $\sigma_{\kappa}$ be the continuous solution of the differential delay problem

$$
\begin{aligned}
u^{\kappa} \sigma_{\kappa}(u) & =\left(2 e^{\gamma}\right)^{\kappa}(\Gamma(\kappa+1))^{-1}, & & 0<u \leq 2, \\
\left(u^{\kappa} \sigma_{\kappa}(u)\right)^{\prime} & =-\kappa u^{-\kappa-1} \sigma_{\kappa}(u-2), & & u>2,
\end{aligned}
$$

where $\gamma$ is Euler's constant and $\Gamma$ is Euler's gamma function.

Let $F_{\kappa}(u)$ and $f_{\kappa}(u)$ be the continuous solutions of the simultaneous differential delay system

$$
\left\{\begin{aligned}
F_{\kappa}(u) & =\frac{1}{\sigma_{\kappa}(u)}, & & 0<u \leq \alpha_{\kappa}, \\
f_{\kappa}(u) & =0, & & 0 \leq u \leq \beta_{\kappa}, \\
\left(u^{\kappa} F_{\kappa}(u)\right)^{\prime} & =\kappa u^{\kappa-1} f_{\kappa}(u-1), & & u>\alpha_{\kappa}, \\
\left(u^{\kappa} f_{\kappa}(u)\right)^{\prime} & =\kappa u^{\kappa-1} F_{\kappa}(u-1), & & u>\beta_{\kappa},
\end{aligned}\right.
$$

where $\alpha_{\kappa}$ and $\beta_{\kappa}$ are real numbers such that

$$
3<\beta_{\kappa}+1<\alpha_{\kappa} .
$$

We note that

(i) $F_{\kappa}(u)$ decreases monotonically toward 1 as $u \rightarrow \infty$,

(ii) $f_{\kappa}(u)$ increases monotonically toward 1 as $u \rightarrow \infty$.

Suppose that $\left\{a_{n}\right\}$ is a (finite) sequence of non-negative real numbers. Then we introduce

$$
\mathcal{A}(d)=\sum_{n \equiv 0} a_{n} .
$$

It is expected that $\frac{\Omega(t)}{t} X$ is a good approximation to $\mathcal{A}(t)$, where $\Omega(t)$ is a multiplicative function satisfying

$$
0 \leq \Omega(p)<\min \{p, c\}
$$

for some constant $c$, and

$$
\prod_{w_{1} \leq p<w}\left(1-\frac{\Omega(p)}{p}\right)^{-1} \leq\left(\frac{\log w}{\log w_{1}}\right)^{3}\left(1+\frac{c_{1}}{\log w_{1}}\right), \quad 2 \leq w_{1}<w,
$$

for some constant $c_{1}>0$. Suppose that there exists a constant $c_{2} \geq 2$ such that

$$
\sum_{t \leq D} \mu^{2}(t) \tau^{2}(t)\left|\mathcal{A}(t)-\frac{\Omega(t)}{t} X\right| \leq c_{2} \frac{X}{(\log X)^{4}} .
$$

Let $\Pi(z)=\prod_{p \leq z} p$ and define

$$
V(x)=\prod_{p \leq x}\left(1-\frac{\Omega(p)}{p}\right) .
$$


Then one has

$$
\sum_{(n, \Pi(z))=1} a_{n} \leq X V(z)\left(F_{\kappa}(s)+O\left(\frac{(\log \log X)^{2}}{(\log X)^{1 / 8}}\right)\right)
$$

and

$$
\sum_{(n, \Pi(z))=1} a_{n} \geq X V(z)\left(f_{\kappa}(s)+O\left(\frac{(\log \log X)^{2}}{(\log X)^{1 / 8}}\right)\right),
$$

where $s=\log D / \log z$.

By (8.1), for $s>\beta_{\kappa}$ we have

$$
s^{\kappa} f_{\kappa}(s)=\int_{\beta_{\kappa}}^{s} \kappa u^{\kappa-1} F_{\kappa}(u-1) d u>F_{\kappa}(s) \int_{\beta_{\kappa}}^{s} \kappa u^{\kappa-1} d u=F_{\kappa}(s)\left(s^{\kappa}-\beta_{\kappa}^{\kappa}\right) .
$$

Therefore one has

$$
\frac{f_{\kappa}(s)}{F_{\kappa}(s)}>1-\left(\frac{\beta_{\kappa}}{s}\right)^{\kappa}
$$

\section{Applications of the switching principle}

Let

$$
\mathcal{A}(t)=\mathcal{A}(t, N):=\mathcal{A}^{(j)}(t, N)=\sum_{\substack{q^{2}+x_{1}^{2}+x_{2}^{2}+x_{3}^{2}=N \\ q \in \mathcal{A}_{j} \\ t \mid x_{1} x_{2} x_{3}}} \omega(q) \omega(\mathbf{x}) .
$$

Note that the function

$$
g(t)=\mu(t) \sum_{\substack{\mathbf{d} \\\left[d_{1}, d_{2}, d_{3}\right]=t \\ d_{j} \mid x_{j}(1 \leq j \leq 3)}} \mu\left(d_{1}\right) \mu\left(d_{2}\right) \mu\left(d_{3}\right)
$$

is a multiplicative function of $t$. If $t$ is square-free and $t \mid x_{1} x_{2} x_{3}$, then $g(t)=1$, and $g(t)=0$ otherwise. Hence for $t$ square-free, we have

$$
\mathcal{A}(t)=\sum_{\substack{q^{2}+x_{1}^{2}+x_{2}^{2}+x_{3}^{2}=N \\ q \in \mathcal{A}_{j} \\ t \mid x_{1} x_{2} x_{3}}} \omega(q) \omega(\mathbf{x}) \mu(t) \sum_{\substack{\mathbf{d} \\\left[d_{1}, d_{2}, d_{3}\right]=t \\ d_{j} \mid x_{j}(1 \leq j \leq 3)}} \mu\left(d_{1}\right) \mu\left(d_{2}\right) \mu\left(d_{3}\right) .
$$

By interchanging the summations, we get

$$
\mathcal{A}(t)=\mu(t) \sum_{\substack{\mathbf{d} \\\left[d_{1}, d_{2}, d_{3}\right]=t}} \mu\left(d_{1}\right) \mu\left(d_{2}\right) \mu\left(d_{3}\right) \mathscr{L}_{\mathbf{d}}(N),
$$

where $\mathscr{L}_{\mathbf{d}}(N)$ is defined in (2.4). The expected main term for $\mathcal{A}(t)$ is

$$
\mathcal{M}_{t}=\mu(t) \sum_{\substack{\mathbf{d} \\\left[d_{1}, d_{2}, d_{3}\right]=t}} \mu\left(d_{1}\right) \mu\left(d_{2}\right) \mu\left(d_{3}\right) \frac{\Sigma_{0}(\mathbf{d}, N) \mathcal{N}_{0}(N)}{d_{1} d_{2} d_{3}},
$$

where $\Sigma_{0}(\mathbf{d}, N)$ and $\mathcal{N}_{0}(N)$ are given in (2.2) and (2.5) respectively. 
Let $X=\Sigma_{0}(\mathbf{e}, N) \mathcal{N}_{0}(N)$ with $\mathbf{e}=(1,1,1)$. We point out that for $N \equiv 4(\bmod 24)$ (cf. [9, (311)]),

$$
1 \ll \Sigma_{0}(\mathbf{e}, N) \ll \log \log N
$$

Therefore we can define

$$
\Omega(t)=t \mu(t) \sum_{\substack{\mathbf{d} \\\left[d_{1}, d_{2}, d_{3}\right]=t}} \mu\left(d_{1}\right) \mu\left(d_{2}\right) \mu\left(d_{3}\right) \frac{\Sigma_{0}(\mathbf{d}, N)}{d_{1} d_{2} d_{3} \Sigma_{0}(\mathbf{e}, N)} .
$$

Then we see that

$$
\mathcal{M}_{t}=\frac{\Omega(t)}{t} X
$$

For $p>2$, we define

$$
\begin{aligned}
& h_{0}(p)= \begin{cases}\frac{1}{p} & \text { if } p \mid N, \\
\frac{-1}{p(p-1)}\left(1+\left(\frac{-N}{p}\right)\right) & \text { if } p \nmid N,\end{cases} \\
& h_{1}(p)= \begin{cases}\frac{-1}{p}\left(\frac{-1}{p}\right) & \text { if } p \mid N, \\
\frac{1}{p-1}\left(\left(\frac{-N}{p}\right)+\frac{1}{p}\left(\frac{-1}{p}\right)\right) & \text { if } p \nmid N,\end{cases} \\
& h_{2}(p)= \begin{cases}\left(\frac{-1}{p}\right) & \text { if } p \mid N, \\
\frac{-1}{p-1}\left(\left(\frac{-1}{p}\right)+\left(\frac{N}{p}\right)\right) & \text { if } p \nmid N,\end{cases} \\
& h_{3}(p)= \begin{cases}-1 & \text { if } p \mid N, \\
\frac{1}{p-1}\left(p\left(\frac{N}{p}\right)+1\right) & \text { if } p \nmid N .\end{cases}
\end{aligned}
$$

The function $\Omega(t)$ is multiplicative with

$$
\Omega(p)=\frac{3\left(1+h_{1}(p)\right)}{1+h_{0}(p)}-\frac{3\left(1+h_{2}(p)\right)}{p\left(1+h_{0}(p)\right)}+\frac{1+h_{3}(p)}{p^{2}\left(1+h_{0}(p)\right)}
$$

for $p>2$ and $\Omega(2)=0$. One can easily show that

$$
0 \leq \Omega(p)<\min \{p, 8\}
$$

and

$$
\Omega(p)=3+O\left(\frac{1}{p}\right) .
$$

Hence (8.2) and (8.3) are established. Now we turn to (8.4). We have

$$
\begin{aligned}
E(D) & :=\sum_{t \leq D} \mu^{2}(t) \tau^{2}(t)\left|\mathcal{A}(t)-\frac{\Omega(t)}{t} X\right| \\
& =\sum_{t \leq D} \mu^{2}(t) \tau^{2}(t) \xi(t, N)\left(\mathcal{A}(t)-\frac{\Omega(t)}{t} X\right),
\end{aligned}
$$

where

$$
\xi(t, N)= \begin{cases}\left|\mathcal{A}(t)-\frac{\Omega(t)}{t} X\right|\left(\mathcal{A}(t)-\frac{\Omega(t)}{t} X\right)^{-1} & \text { if } \mathcal{A}(t)-\frac{\Omega(t)}{t} X \text { is non-zero } \\ 0 & \text { otherwise. }\end{cases}
$$


Recalling (9.1), (9.2) and (9.3), we have

$$
E(D)=\sum_{\substack{d_{1}, d_{2}, d_{3} \\\left[d_{1}, d_{2}, d_{3}\right] \leq D}} \beta\left(d_{1}, d_{2}, d_{3}\right)\left(\mathscr{L}_{\mathbf{d}}(N)-\frac{\Sigma_{0}(\mathbf{d}, N) \mathcal{N}_{0}(N)}{d_{1} d_{2} d_{3}}\right),
$$

where

$$
\beta\left(d_{1}, d_{2}, d_{3}\right)=\mu\left(d_{1}\right) \mu\left(d_{2}\right) \mu\left(d_{3}\right) \mu\left(\left[d_{1}, d_{2}, d_{3}\right]\right) \tau^{2}\left(\left[d_{1}, d_{2}, d_{3}\right]\right) \xi\left(\left[d_{1}, d_{2}, d_{3}\right], N\right) .
$$

Obviously,

$$
\beta\left(d_{1}, d_{2}, d_{3}\right) \ll \prod_{j=1}^{3} \tau^{2}\left(d_{j}\right) .
$$

Invoking Proposition 3, we see that (8.4) holds true with $D=P^{1 / 2-\varepsilon}$. Therefore the inequalities (8.5) and (8.6) hold for the sequence

$$
a_{n}:=a_{n}^{(j)}=\sum_{\substack{q^{2}+x_{1}^{2}+x_{2}^{2}+x_{3}^{2}=N \\ q \in \mathcal{A}_{j} \\ x_{1} x_{2} x_{3}=n}} \omega(q) \omega(\mathbf{x}) .
$$

Lemma 16. Let $c_{1}(t)=1$ or $c_{1}(t)=0$ according to $t \geq 1$ or $t<1$. We define $c_{j}(t)$ inductively by

$$
c_{j}(t)=\int_{j}^{\max (j, t)} \frac{c_{j-1}(x-1)}{x-1} d x .
$$

Then for $1 \leq j \leq 15$, one has

$$
\frac{N}{\log N} \ll \mathcal{N}_{0}^{j}(N) \ll \frac{N}{\log N}
$$

and

$$
\mathcal{N}_{0}^{j}(N)=\left(c_{j}(16)+O\left(\frac{1}{\log N}\right)\right) \mathcal{N}_{0}^{1}(N)
$$

Proof. In view of [2, (2.16)], we have

$$
C_{j}(x)=c_{j}(16)+O\left(\frac{1}{\log N}\right) \text { for } \frac{P}{2}<x<P .
$$

Hence

$$
\mathcal{N}_{0}^{j}(N)-c_{j}(16) \mathcal{N}_{0}^{1}(N) \ll \frac{N}{\log ^{2} N} .
$$

We get the desired results by observing that

$$
\frac{N}{\log N} \ll \mathcal{N}_{0}^{1}(N) \ll \frac{N}{\log N} .
$$

Remark. We record some numerical values:

$$
c_{2}(16)>2.70805, \quad c_{3}(16)>2.912112, \quad c_{4}(16)>1.663428, \quad c_{5}(16)>0.563668 .
$$


Proof of Theorem 1.1. Our objective is to prove

$$
R(N):=\sum_{\substack{p^{2}+x_{1}^{2}+x_{2}^{2}+x_{3}^{2}=N \\ x_{1}, x_{2}, x_{3} \in \bigcup_{j=1}^{5} \mathcal{A}_{j}}} \omega(p) \omega(\mathbf{x})>0 .
$$

Observe that

$$
\begin{aligned}
& \sum_{p \in \mathcal{A}_{1}}=\sum_{p \in \mathcal{A}_{1}}-\sum_{p \in \mathcal{A}_{1}}-\sum_{p \in \mathcal{A}_{1}}-\sum_{p \in \mathcal{A}_{1}} \\
& x_{1}, x_{2}, x_{3} \in \bigcup_{j=1}^{5} \mathcal{A}_{j} \quad x_{1}, x_{2}, x_{3} \in \bigcup_{j=1}^{15} \mathcal{A}_{j} \quad x_{1} \in \bigcup_{j=6}^{15} \mathcal{A}_{j} \quad x_{1} \in \bigcup_{j=1}^{5} \mathcal{A}_{j} \quad x_{1}, x_{2} \in \bigcup_{j=1}^{5} \mathcal{A}_{j} \\
& x_{2}, x_{3} \in \bigcup_{j=1}^{15} \mathcal{A}_{j} \quad x_{2} \in \bigcup_{j=6}^{15} \mathcal{A}_{j} \quad x_{3} \in \bigcup_{j=6}^{15} \mathcal{A}_{j} \\
& x_{3} \in \bigcup_{j=1}^{15} \mathscr{A}_{j} \\
& \geq \sum_{\substack{p \in \mathcal{A}_{1} \\
x_{1}, x_{2}, x_{3} \in \bigcup_{j=1}^{15} \mathcal{A}_{j}}}-\sum_{\substack{q \in \bigcup_{j=1}^{15} \mathcal{A}_{j} \\
x_{1} \in \bigcup_{j=6}^{15} \mathcal{A}_{j} \\
x_{2}, x_{3} \in \bigcup_{j=1}^{15} \mathcal{A}_{j}}}-\sum_{\substack{q \in \bigcup_{j=1}^{15} \mathcal{A}_{j} \\
x_{2} \in \bigcup_{j=6}^{15} \mathcal{A}_{j}}} \sum_{\substack{x_{1}, x_{3} \in \bigcup_{j=1}^{15} \mathcal{A}_{j} \\
q \in \bigcup_{j=1}^{15} \mathcal{A}_{j} \\
x_{3} \in \bigcup_{j=6}^{15} \mathcal{A}_{j} \\
x_{1}, x_{2} \in \bigcup_{j=1}^{15} \mathcal{A}_{j}}}
\end{aligned}
$$

By switching the roles of $q$ and $x_{j}$, we obtain

$$
R(N) \geq \sum_{\substack{p^{2}+x_{1}^{2}+x_{2}^{2}+x_{3}^{2}=N \\ x_{1}, x_{2}, x_{3} \in \bigcup_{j=1}^{15} \mathcal{A}_{j}}} \omega(p) \omega(\mathbf{x})-3 \sum_{\substack{q^{2}+x_{1}^{2}+x_{2}^{2}+x_{3}^{2}=N \\ q \in \bigcup_{j=6}^{15} \mathcal{A}_{j} \\ x_{1}, x_{2}, x_{3} \in \bigcup_{j=1}^{15} \mathcal{A}_{j}}} \omega(q) \omega(\mathbf{x}) .
$$

In view of the three-dimensional sieve, we have

$$
\begin{aligned}
\sum_{\substack{p^{2}+x_{1}^{2}+x_{2}^{2}+x_{3}^{2}=N \\
x_{1}, x_{2}, x_{3} \in \bigcup_{j=1}^{15} \mathcal{A}_{j}}} \omega(p) \omega(\mathbf{x}) & =\sum_{(n, \Pi(z))=1} \sum_{\substack{p^{2}+x_{1}^{2}+x_{2}^{2}+x_{3}^{2}=N \\
x_{1} x_{2} x_{3}=n}} \omega(p) \omega(\mathbf{x}) \\
& \geq\left(f_{3}(8-\varepsilon)-\varepsilon\right) \Sigma_{0}(\mathbf{e}, N) \mathcal{N}_{0}^{1}(N) V(z),
\end{aligned}
$$

where $z=P^{1 / 16}$. Similarly,

$$
\begin{aligned}
\sum_{\substack{q^{2}+x_{1}^{2}+x_{2}^{2}+x_{3}^{2}=N \\
q \in \bigcup_{j=6}^{15} \mathcal{A}_{j} \\
x_{1}, x_{2}, x_{3} \in \bigcup_{j=1}^{15} \mathcal{A}_{j}}} \omega(q) \omega(\mathbf{x}) & =\sum_{j=6}^{15} \sum_{\substack{(n, \Pi(z))=1 \\
x_{1} x_{2} x_{3}=n \\
q^{2}+x_{1}^{2}+x_{2}^{2}+x_{3}^{2}=N}} \omega(q) \omega(\mathbf{x}) \\
& \leq\left(F_{3}(8-\varepsilon)+\varepsilon\right) \Sigma_{0}(\mathbf{e}, N) \sum_{j=6}^{15} \mathcal{N}_{0}^{j}(N) V(z) .
\end{aligned}
$$

By Lemma 16, we obtain

$$
\sum_{\substack{q^{2}+x_{1}^{2}+x_{2}^{2}+x_{3}^{2}=N \\ q \in \bigcup_{j=6}^{15} \mathcal{A}_{j} \\ x_{1}, x_{2}, x_{3} \in \bigcup_{j=1}^{15} \mathcal{A}_{j}}} \omega(q) \omega(\mathbf{x}) \leq\left(F_{3}(8-\varepsilon) \sum_{j=6}^{15} c_{j}(16)+\varepsilon\right) \Sigma_{0}(\mathbf{e}, N) \mathcal{N}_{0}^{1}(N) V(z) .
$$


Therefore

$$
\begin{aligned}
R(N) & \geq\left(f_{3}\left(\frac{16}{2}\right)-3 F_{3}\left(\frac{16}{2}\right) \sum_{j=6}^{15} c_{j}(16)-\varepsilon\right) \Sigma_{0}(\mathbf{e}, N) \mathcal{N}_{0}^{1}(N) V(z) \\
& =\left(\frac{f_{3}(8)}{3 F_{3}(8)}-\sum_{j=6}^{15} c_{j}(16)-\varepsilon\right) 3 F_{3}(8) \Sigma_{0}(\mathbf{e}, N) \mathcal{N}_{0}^{1}(N) V(z) \\
& =\left(C_{0}-\varepsilon\right) 3 F_{3}(8) \Sigma_{0}(\mathbf{e}, N) \mathcal{N}_{0}^{1}(N) V(z)
\end{aligned}
$$

where

$$
C_{0}=\frac{f_{3}(8)}{3 F_{3}(8)}-\sum_{j=6}^{15} c_{j}(16)
$$

By (8.7), we have

$$
\frac{f_{3}(8)}{F_{3}(8)}>1-\left(\frac{\beta_{3}}{8}\right)^{3}
$$

where $\beta_{3} \leq 6.640859$. Thus

$$
C_{0}>\frac{1}{3}\left(1-\left(\frac{\beta_{3}}{8}\right)^{3}\right)-\sum_{j=6}^{15} c_{j}(16) .
$$

Now numerical computations reveal that $C_{0}>0.003$. Thus Theorem 1.1 is established.

One can do numerical computations in the following way. We have

$$
C_{0}>\frac{1}{3}\left(1-\left(\frac{\beta_{3}}{8}\right)^{3}\right)+\sum_{j=1}^{5} c_{j}(16)-\sum_{j=1}^{15} c_{j}(16) .
$$

Brüdern and Kawada pointed out that as a consequence of linear sieve, one has (see [2, (6.35)])

$$
\sum_{j=1}^{15} c_{j}(16) \leq 16 e^{-\gamma} F_{1}(16) \leq 16 e^{-\gamma}\left(1+10^{-9}\right) .
$$

Therefore,

$$
C_{0}>\frac{1}{3}\left(1-\left(\frac{\beta_{3}}{8}\right)^{3}\right)+\sum_{j=1}^{5} c_{j}(16)-16 e^{-\gamma}\left(1+10^{-9}\right) .
$$

Then we actually need the numerical values of $c_{j}(16)$ for $1 \leq j \leq 5$.

Proof of Theorem 1.2. The proof is as same as Theorem 1.1 except that we consider

$$
R^{\prime}(N):=\sum_{\substack{q^{2}+x_{1}^{2}+x_{2}^{2}+x_{3}^{2}=N \\ q, x_{1}, x_{2}, x_{3} \in \bigcup_{j=1}^{4} \mathcal{A}_{j}}} \omega(q) \omega(\mathbf{x})
$$


In view of the switching principle and the three-dimensional sieve, the lower bound for $R^{\prime}(N)$ is

$$
\begin{aligned}
R^{\prime}(N) & \geq\left(f_{3}(8) \sum_{j=1}^{4} c_{j}(16)-3 F_{3}(8) \sum_{j=5}^{15} c_{j}(16)-\varepsilon\right) \Sigma_{0}(\mathbf{e}, N) \mathcal{N}_{0}^{1}(N) V(z) \\
& =\left(\frac{f_{3}(8)}{3 F_{3}(8)} \sum_{j=1}^{4} c_{j}(16)-\sum_{j=5}^{15} c_{j}(16)-\varepsilon\right) 3 F_{3}(8) \Sigma_{0}(\mathbf{e}, N) \mathcal{N}_{0}^{1}(N) V(z) \\
& =\left(C_{0}^{\prime}-\varepsilon\right) 3 F_{3}(8) \Sigma_{0}(\mathbf{e}, N) \mathcal{N}_{0}^{1}(N) V(z),
\end{aligned}
$$

where

$$
C_{0}^{\prime}=\frac{f_{3}(8)}{3 F_{3}(8)} \sum_{j=1}^{4} c_{j}(16)-\sum_{j=5}^{15} c_{j}(16)
$$

Similarly, we have

$$
C_{0}^{\prime}>\frac{1}{3}\left(1-\left(\frac{\beta_{3}}{8}\right)^{3}\right) \sum_{j=1}^{4} c_{j}(16)+\sum_{j=1}^{4} c_{j}(16)-16 e^{-\gamma}\left(1+10^{-9}\right) .
$$

Again a numerical computation reveals that $C_{0}^{\prime}>0$. We point out that we gain $P_{4}$ instead of $P_{5}$ due to the constant $\sum_{j=1}^{4} c_{j}(16)$ in place of 1 . This completes the proof of Theorem 1.2.

Acknowledgement. The authors wish to thank Professors Jörg Brüdern and Maosheng Xiong for their helpful comments. The authors also wish to thank Dr. Yuk-Kam Lau for his many helpful discussions.

\section{References}

[1] J. Brüdern and E. Fouvry, Lagrange's four squares theorem with almost prime variables, J. reine angew. Math. 454 (1994), 59-96.

[2] J. Brüdern and K. Kawada, Ternary problems in additive prime number theory, in: Analytic number theory, Dev. Math. 6, Kluwer Academic Publishers, Dordrecht (2002), 39-91.

[3] Y. Cai, Lagrange's four squares theorem with variables of special type, Int. J. Number Theory 6 (2010), 1801-1817.

[4] J.R. Chen, On the representation of a large even integer as the sum of a prime and the product of at most two primes, Sci. Sin. 16 (1973), 157-176.

[5] T. Estermann, A new application of the Hardy-Littlewood-Kloosterman method, Proc. Lond. Math. Soc. (3) 12 (1962), 425-444.

[6] G. Greaves, On the representation of a number in the form $x^{2}+y^{2}+p^{2}+q^{2}$ where $p, q$ are odd primes, Acta Arith. 29 (1976), 257-274.

[7] H. Halberstam and H. E. Richert, Sieve methods, London Math. Soc. Monogr. 4, Academic Press, London 1974.

[8] D. R. Heath-Brown, The square sieve and consectutive square-free numbers, Math. Ann. 226 (1984), 251-259.

[9] D. R. Heath-Brown and D. I. Tolev, Lagrange's four squares theorem with one prime and three almost prime variables, J. reine angew. Math. 558 (2003), 159-224.

[10] L. K. Hua, Some results in the additive prime number theory, Quart. J. Math. Oxford 9 (1938), 68-80.

[11] H. D. Kloosterman, On the representation of numbers in the form $a x^{2}+b y^{2}+c z^{2}+d t^{2}$, Acta Math. 49 (1926), 407-466.

[12] V. A. Plaksin, An asymptotic formula for the number of solutions of a nonlinear equation for prime numbers, Math. USSR Izv. 18 (1982), 275-348. 
[13] E. V. Podsypanin, On the representation of the integer by positive quadratic forms with square-free variables, Acta Arith. 27 (1975), 459-488.

[14] P. Shields, Some applications of the sieve methods in number theory, PhD thesis, University of Wales, 1979.

[15] D. I. Tolev, Lagrange's four squares theorem with variables of special type, in: Proceedings of the session in analytic number theory and Diophantine equations (Bonn 2002), Bonner Math. Schriften 360, University of Bonn, Bonn (2003), 159-224.

[16] R. C. Vaughan, The Hardy-Littlewood method, 2nd ed., Cambridge University Press, Cambridge 1997.

Kai-Man Tsang, Department of Mathematics, The University of Hong Kong, Porfulam Road, Hong Kong e-mail: kmtsang@maths.hku.hk

Lilu Zhao, School of Mathematics, Hefei University of Technology, Hefei, 230009, China e-mail: zhaolilu@gmail.com

Eingegangen 1. Oktober 2013 